\title{
Evaluation of the Effectiveness of Digital Technology Interventions to Reduce Loneliness in Older Adults: Systematic Review and Meta-analysis
}

Syed Ghulam Sarwar Shah ${ }^{1,2}$, MBBS, MA, MSc, PhD; David Nogueras ${ }^{3}$, MBA; Hugo Cornelis van Woerden ${ }^{4,5,6}$, MBChB, MPH, PhD, FFPH; Vasiliki Kiparoglou ${ }^{1,7}$, BSc, MSc, MBA, PhD

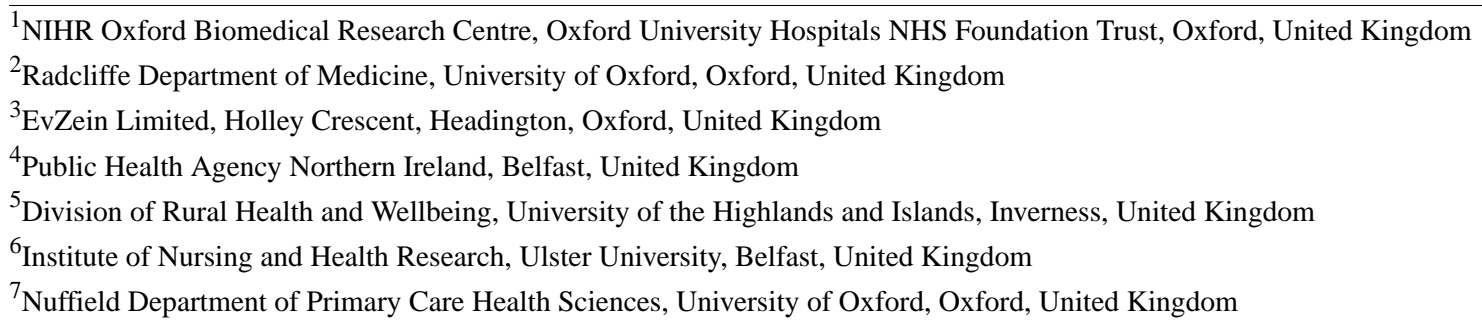

\section{Corresponding Author:}

Syed Ghulam Sarwar Shah, MBBS, MA, MSc, PhD

NIHR Oxford Biomedical Research Centre

Oxford University Hospitals NHS Foundation Trust

John Radcliffe Hospital

Oxford, OX3 9DU

United Kingdom

Phone: 441865221262

Email: sarwar.shah@ouh.nhs.uk

\section{Abstract}

Background: Loneliness is a serious public health issue, and its burden is increasing in many countries. Loneliness affects social, physical, and mental health, and it is associated with multimorbidity and premature mortality. In addition to social interventions, a range of digital technology interventions (DTIs) are being used to tackle loneliness. However, there is limited evidence on the effectiveness of DTIs in reducing loneliness, especially in adults. The effectiveness of DTIs in reducing loneliness needs to be systematically assessed.

Objective: The objective of this study is to assess the effectiveness of DTIs in reducing loneliness in older adults.

Methods: We conducted electronic searches in PubMed, MEDLINE, CINAHL, Embase, and Web of Science for empirical studies published in English from January 1, 2010, to July 31, 2019. The study selection criteria included interventional studies that used any type of DTIs to reduce loneliness in adults (aged $\geq 18$ years) with a minimum intervention duration of 3 months and follow-up measurements at least 3 months after the intervention. Two researchers independently screened articles and extracted data using the PICO (participant, intervention, comparator, and outcome) framework. The primary outcome measure was loneliness. Loneliness scores in both the intervention and control groups at baseline and at follow-up at 3, 4, 6, and 12 months after the intervention were extracted. Data were analyzed via narrative synthesis and meta-analysis using RevMan (The Cochrane Collaboration) software.

Results: A total of 6 studies were selected from 4939 screened articles. These studies included 1 before and after study and 5 clinical trials (4 randomized clinical trials and 1 quasi-experimental study). All of these studies enrolled a total of 646 participants (men: $\mathrm{n}=154,23.8 \%$; women: $\mathrm{n}=427,66.1 \%$; no gender information: $\mathrm{n}=65,10.1 \%$ ) with an average age of 73-78 years (SD 6-11). Five clinical trials were included in the meta-analysis, and by using the random effects model, standardized mean differences (SMDs) were calculated for each trial and pooled across studies at the 3-, 4-, and 6-month follow-ups. The overall effect estimates showed no statistically significant difference in the effectiveness of DTIs compared with that of usual care or non-DTIs at follow-up at 3 months (SMD 0.02; 95\% CI -0.36 to $0.40 ; P=.92$ ), 4 months (SMD $-1.11 ; 95 \% \mathrm{CI}-2.60$ to $0.38 ; P=.14$ ), and 6 months (SMD $-0.11 ; 95 \% \mathrm{CI}-0.54$ to $0.32 ; P=.61$ ). The quality of evidence was very low to moderate in these trials. 
Conclusions: Our meta-analysis shows no evidence supporting the effectiveness of DTIs in reducing loneliness in older adults. Future research may consider randomized controlled trials with larger sample sizes and longer durations for both the interventions and follow-ups.

International Registered Report Identifier (IRRID)： RR2-10.1136/bmjopen-2019-032455

(J Med Internet Res 2021;23(6):e24712) doi: 10.2196/24712

\section{KEYWORDS}

loneliness; older people; digital technology; effectiveness; efficacy; evidence; systematic review; meta-analysis

\section{Introduction}

\section{Background}

Loneliness is a multifaceted public health problem [1]. The burden of loneliness is high in some countries [2-9], and it is increasing in many other countries [10]. Loneliness is expected to rise because of lockdowns, quarantine, self-isolation, and social distancing measures that are being enforced in several countries to tackle the COVID-19 pandemic $[11,12]$. Therefore, tackling loneliness is imperative, and digital technology could play a major role in addressing loneliness [13].

Loneliness refers to an individual's subjective feelings of a perceived discrepancy between actual and desired social relationships $[14,15]$. Although loneliness affects people of all ages $[15,16]$, older, younger, and vulnerable people are affected more by it $[7,17,18]$. Risk factors of loneliness include demographic characteristics, social factors, and physical environments [17-19]. Loneliness enhances the risk of poor physical and mental health [14,20-23], dementia [24], premature mortality, and all-cause mortality [21], particularly in older adults [23]. In addition, the implications of loneliness include the high costs of health and well-being (eg, between $£ 6429.00$ [US \$8074.80] and £9616.00 [US \$12,077.70] per person per year in the United Kingdom) [25] as well as lost work days and productivity (eg, costing up to $£ 2.5$ billion [US $\$ 3.14$ billion] per annum for employers in the United Kingdom) [26]. Therefore, it is imperative to tackle loneliness.

Loneliness is being addressed through a range of social [27] and technological interventions [28]. The latter type of interventions includes numerous and diverse types of digital apps, web-based social networking tools, sensors, and robots [29]. Although these tools use digital technology, they are heterogeneous in many aspects, including the means they provide to socially connect; the purposes for which they are used; the ways and methods of their application; the frequency of their use; and their users, who differ from each other in many traits such as demographic, social, and economic characteristics, and some may have physical and mental limitations. Therefore, these digital technology tools need to be systematically evaluated for their effectiveness in tackling loneliness.

Several published reviews have reported that digital technology interventions (DTIs) are effective in reducing loneliness [30-34]. However, some of these studies are weak and have a high risk of bias [35], and other studies have used a few selected technological interventions and covered literature published over a short span, such as the 3-year period from January 2010 to January 2013 [31].
There is limited evidence on the effectiveness of DTIs for loneliness [36], and there are calls for further research [32,33] to assess and identify the latest DTIs that are effective in reducing loneliness [34,36]. In addition, evaluation of the latest evidence on the effectiveness of DTIs in reducing loneliness is imperative from the perspectives of patients and their families and other stakeholders such as health and social care providers and health insurers [37].

\section{Study Objectives}

The primary objective of this study is to assess the effectiveness of DTIs in reducing loneliness in adults. The secondary objective is to identify DTIs that are used to reduce loneliness in adults.

\section{Review Questions}

The main research question was "Are DTIs effective for reducing loneliness in adults?" The secondary question was "What DTIs are used for reducing loneliness in adults?"

\section{Outcome Measures}

The main outcome measure was loneliness. We extracted data on loneliness measured at both the baseline (before the intervention) and follow-ups (at least 3 months after the intervention) for the intervention groups and control groups, if any.

\section{Methods}

\section{Study Design, Conduct, and Reporting}

We undertook a systematic review and meta-analysis as suggested in the Cochrane Methods for Systematic Reviews of Interventions [38]. We have reported the findings in accordance with the PRISMA (Preferred Reporting Items for Systematic Reviews and Meta-Analyses) guidelines [39].

\section{Protocol Registration and Publication}

We registered this systematic review and meta-analysis with the PROSPERO database on June 10, 2019 (registration ID: CRD42019131524) [40], and we published our protocol [37] before undertaking this study.

\section{Patient and Public Involvement}

A patient and public manager affiliated with our research center reviewed the study protocol and provided suggestions that were incorporated into the protocol. We had no access to any patient diagnosed with loneliness; therefore, we could not include any patients or members of the public in the design and conduct of the study. However, the findings of this study will be disseminated as an open access publication that will be freely available to patients and everyone else globally. 


\section{Definition of DTI}

We define the term DTI as an intervention that applies digital technology, that is, the technology, equipment, and apps that process information in the form of numeric codes, usually a binary code [41].

\section{Eligibility Criteria}

We selected studies that met our predefined eligibility criteria [37]. Study designs included interventional studies (randomized and nonrandomized) that investigated the effects of DTIs on loneliness. We included a range of DTIs, that is, computers, computer tablets, iPads, internet, web-based videos, communication, chatting, social groups, meetings, conferences and messages, sensors, social robots, smart mobile phones, social media tools, and the World Wide Web. We set 3 months as the minimum intervention duration and follow-up period. The research participants were adults, both male and female, aged 18 years or more. We included different settings, that is, residential dwellings, including private residences and care or nursing homes or centers in any country. The studies were limited to journal articles in English published from January 1, 2010, to July 31, 2019.

\section{Information Sources and Keywords}

We electronically searched PubMed, MEDLINE, CINAHL, Embase, and Web of Science and covered the publication period from January 1, 2010, to July 31, 2019. We used an a priori list of keywords prepared in our preliminary literature searches [37]. The keywords were of 2 categories: medical condition or problem (ie, loneliness, lonely, isolation, aloneness, disconnect*, solitude, singleness*, lonesomeness, solitariness, and remoteness) and intervention or technology (ie, digital, technolog*, sensor*, robot*, internet, social media, *phone*, online, iPad ${ }^{*}$, tablet $^{*}$, computer $^{*}$, electronic, web, video, and videoconference), as reported in our published protocol [37].

\section{Literature Searches}

First, we searched the keywords in the subject headings such as MeSH major terms in PubMed or equivalent terms in other databases (for detailed search history, see Multimedia Appendix 1). Thereafter, we searched for keywords in the title and abstract fields in the selected databases using 3 Boolean operators: "OR," "AND," and "NOT." In addition, we hand searched the reference lists of the shortlisted articles. We wrote emails to the authors of 2 studies requesting for full copies of their research articles $[42,43]$, which were gratefully emailed to us. We contacted the authors of 2 further studies for missing or additional data $[44,45]$. We had a good response from the authors of both studies, and data were thankfully provided for 1 study only [45]. We sought support from an expert librarian at our library for running literature searches.

\section{Study Selection}

Literature searches retrieved 4939 articles, of which 965 duplicate articles were removed (Figure 1). Two researchers (SGSS and DN) independently screened the remaining articles $(n=3974)$ by title, which was followed by reading the abstracts of 442 articles (Figure 1). This screening process led to the exclusion of 3876 articles and identification of 98 articles for full-text review. Three researchers (SGSS, DN, and VK) independently read the full texts of these 98 articles.

When recommendations differed between reviewers at the title, abstract, and full-text review stages, another reviewer $(\mathrm{HCvW})$ reviewed these articles, and his recommendations to either include or exclude an article were final.

Finally, 92 articles were excluded, and the remaining 6 articles were included in the data extraction (Figure 1). All these 6 studies were included in the narrative synthesis, whereas 5 studies-all clinical trials involving an intervention group and a control group-were included in the meta-analysis (Figure 1). One study with a pre- and postintervention design involving only the intervention group was excluded from the meta-analysis. 
Figure 1. PRISMA (Preferred Reporting Items for Systematic Reviews and Meta-Analyses) study selection flow diagram.
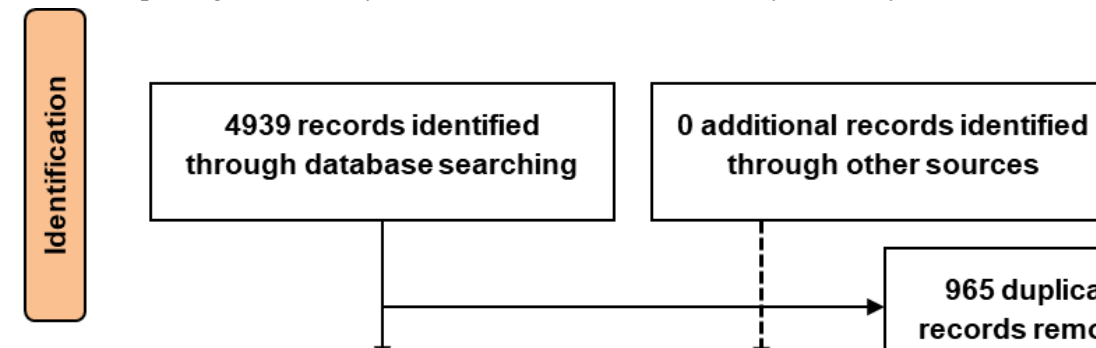

3974 records after duplicates removed
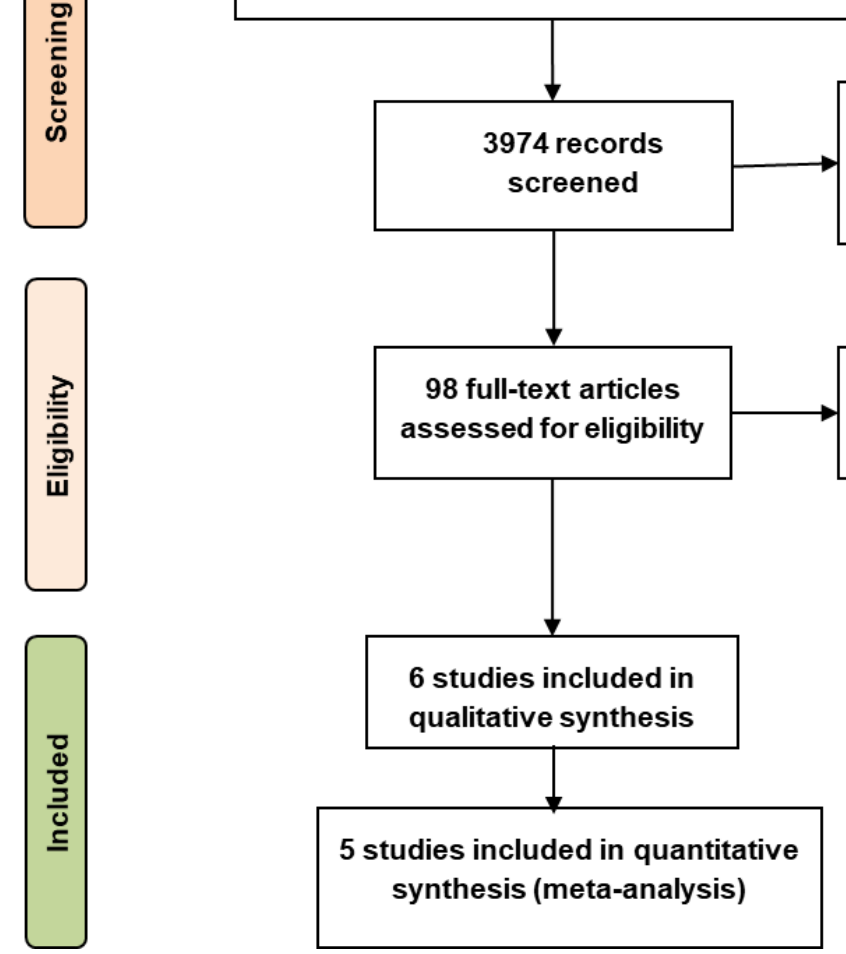

3876 records excluded (title screening $=3532$, abstract review=344)
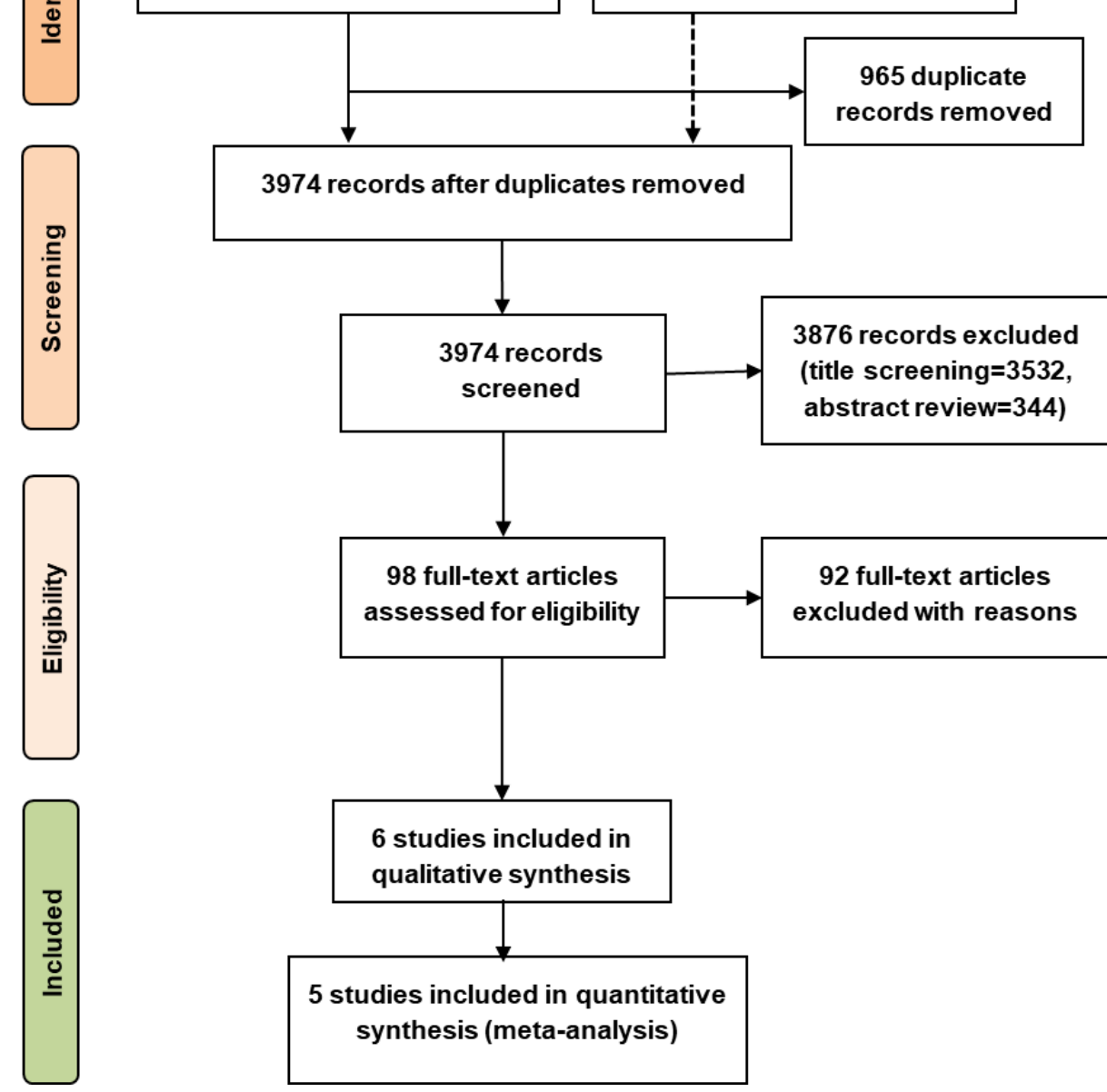

\section{Data Collection Process}

For data collection, we used an a priori data extraction template (Tables 1 and 2), which comprised several columns: authors, year, and country of study; study aim or objectives; research design; settings; participants' characteristics (age, gender, and ethnicity); health or medical condition; sampling method and sample size; participant attrition (numbers and percentages);

research methods and data collection tools; interventions (eg, type and tool of digital technology); comparators (eg, alternative intervention, placebo, or care as usual); intervention duration (weeks or months); measurement stages (eg, baseline and follow-up: weeks or months after the baseline); outcomes, result, and findings (eg, loneliness scores, including statistics; eg, mean values, SDs, SEs, and CIs); and study authors' conclusions [37]. 
Table 1. Characteristics of included studies, participants, sampling methods and sizes, and data collection tools.

\begin{tabular}{|c|c|c|c|c|c|c|c|c|c|c|c|c|c|}
\hline \multirow{2}{*}{$\begin{array}{l}\text { Study, } \\
\text { coun- } \\
\text { try }\end{array}$} & \multirow{2}{*}{$\begin{array}{l}\text { Quality } \\
\text { of evi- } \\
\text { dence }^{\mathrm{a}} \\
\text { (review- } \\
\text { ers' as- } \\
\text { sess- } \\
\text { ment) }\end{array}$} & \multirow{2}{*}{$\begin{array}{l}\text { Research } \\
\text { design }\end{array}$} & \multirow[t]{2}{*}{ Settings } & \multicolumn{3}{|c|}{ Participants } & \multirow{2}{*}{$\begin{array}{l}\text { Main } \\
\text { health } \\
\text { or medi- } \\
\text { cal con- } \\
\text { ditions } \\
\text { investi- } \\
\text { gated }\end{array}$} & \multirow{2}{*}{$\begin{array}{l}\text { Sam- } \\
\text { pling } \\
\text { method }\end{array}$} & \multicolumn{3}{|c|}{ Sample size } & \multirow{2}{*}{$\begin{array}{l}\text { Partici- } \\
\text { pant attri- } \\
\text { tion }\end{array}$} & \multirow{2}{*}{$\begin{array}{l}\text { Re- } \\
\text { search } \\
\text { methods } \\
\text { or data } \\
\text { collec- } \\
\text { tion } \\
\text { tools } \\
\text { Loneli- } \\
\text { ness } \\
\text { scale } \\
\text { used }\end{array}$} \\
\hline & & & & $\begin{array}{l}\text { Age } \\
\text { (years) }\end{array}$ & Gender & Ethnicity & & & Total & $\begin{array}{l}\text { Inter- } \\
\text { ven- } \\
\text { tion } \\
\text { group }\end{array}$ & $\begin{array}{l}\text { Con- } \\
\text { trol } \\
\text { group }\end{array}$ & & \\
\hline $\begin{array}{l}\text { Tsai et } \\
\text { al } \\
(2010) \\
{[46],} \\
\text { Tai- } \\
\text { wan }\end{array}$ & Medium & $\begin{array}{l}\text { Quasi-ex- } \\
\text { perimen- } \\
\text { tal study } \\
\left(\mathrm{NRCT}^{\mathrm{b}}\right)\end{array}$ & $\begin{array}{l}\text { Nursing } \\
\text { home }\end{array}$ & $\begin{array}{l}\text { Base- } \\
\text { line: ex- } \\
\text { perimen- } \\
\text { tal } \\
\text { group: } \\
\text { average } \\
\text { age } 74.2 \\
\text { (SD } \\
\text { 10.18); } \\
\text { control } \\
\text { group: } \\
\text { average } \\
\text { age } \\
78.48 \\
\text { (SD } \\
6.75)\end{array}$ & $\begin{array}{l}\text { Male=24 } \\
\text { (experimen- } \\
\text { tal } \\
\text { group=10; } \\
\text { control } \\
\text { group=14); } \\
\text { female=33 } \\
(\text { experimen- } \\
\text { tal } \\
\text { group=14; } \\
\text { control } \\
\text { group=19) }\end{array}$ & $\begin{array}{l}\text { Not report- } \\
\text { ed (proba- } \\
\text { bly all Tai- } \\
\text { wanese or } \\
\text { Chinese) }\end{array}$ & $\begin{array}{l}\text { Loneli- } \\
\text { ness and } \\
\text { depres- } \\
\text { sion }\end{array}$ & $\begin{array}{l}\text { Purpo- } \\
\text { sive }\end{array}$ & $\begin{array}{l}57 \text { base- } \\
\text { line; } 49 \\
\text { end of } \\
\text { study }\end{array}$ & $\begin{array}{l}24 \\
\text { base- } \\
\text { line; } \\
21 \text { fol- } \\
\text { low- } \\
\text { up }\end{array}$ & $\begin{array}{l}33 \\
\text { base- } \\
\text { line; } \\
28 \text { fol- } \\
\text { low- } \\
\text { up }\end{array}$ & $\begin{array}{l}8 \text { ( } 5 \text { from } \\
\text { control } \\
\text { group and } \\
3 \text { from } \\
\text { experi- } \\
\text { mental } \\
\text { group); } \\
\text { attrition } \\
\text { rate }=14 \%\end{array}$ & $\begin{array}{l}\text { UCLA }^{c} \\
\text { loneli- } \\
\text { ness } \\
\text { scale } \\
{[47]}\end{array}$ \\
\hline $\begin{array}{l}\text { van } \\
\text { der } \\
\text { Heide } \\
\text { et al } \\
(2012) \\
{[48] \text {, }} \\
\text { The } \\
\text { Nether- } \\
\text { lands }\end{array}$ & Low & $\begin{array}{l}\text { Before } \\
\text { and after } \\
\text { study } \\
\text { (with in- } \\
\text { terven- } \\
\text { tion } \\
\text { group on- } \\
\text { ly, no } \\
\text { control } \\
\text { group) }\end{array}$ & $\begin{array}{l}\text { Older } \\
\text { home } \\
\text { care }\end{array}$ & $\begin{array}{l}\text { Base- } \\
\text { line: av- } \\
\text { erage } \\
\text { age } 73.2 \\
\text { (SD } \\
11.8 \text { ), } \\
\text { range } \\
32-90 \text {; } \\
\text { end of } \\
\text { study: } \\
\text { average } \\
\text { age } 73.1 \\
\text { (SD } \\
11.2) \text { ), } \\
\text { range } \\
38-90\end{array}$ & $\begin{array}{l}\text { Baseline: } \\
\text { male=26 } \\
(30.2 \%), \\
\text { female=60 } \\
(69.8 \%), \\
\text { missing } \\
\text { values=44; } \\
\text { end of } \\
\text { study: } \\
\text { male=25 } \\
(29.4 \%), \\
\text { female=60 } \\
(70.6 \%), \\
\text { missing } \\
\text { values=0 }\end{array}$ & $\begin{array}{l}\text { Not report- } \\
\text { ed }\end{array}$ & $\begin{array}{l}\text { Loneli- } \\
\text { ness and } \\
\text { safety } \\
\text { issues }\end{array}$ & $\begin{array}{l}\text { Conve- } \\
\text { nience }\end{array}$ & 130 & 130 & $\begin{array}{l}85=\text { in- } \\
\text { terven- } \\
\text { tion } \\
\text { group } \\
\text { at the } \\
\text { end of } \\
\text { study; } \\
\text { no } \\
\text { con- } \\
\text { trol } \\
\text { group }\end{array}$ & $\begin{array}{l}45 ; \text { attri- } \\
\text { tion } \\
\text { rate= }=34.6 \%\end{array}$ & $\begin{array}{l}\text { De } \\
\text { Jong- } \\
\text { Gierveld } \\
\text { loneli- } \\
\text { ness } \\
\text { scale } \\
\text { (score } \\
\text { range: } \\
0-11) \\
{[49]}\end{array}$ \\
\hline
\end{tabular}




\begin{tabular}{|c|c|c|c|c|c|c|c|c|c|c|c|c|c|}
\hline $\begin{array}{l}\text { Study, } \\
\text { coun- } \\
\text { try }\end{array}$ & $\begin{array}{l}\text { Quality } \\
\text { of evi- } \\
\text { dence }^{\mathrm{a}} \\
\text { (review- } \text { ers' as- } \\
\text { sess- } \\
\text { ment) }\end{array}$ & $\begin{array}{l}\text { Research } \\
\text { design }\end{array}$ & Settings & \multicolumn{3}{|c|}{ Participants } & $\begin{array}{l}\text { Main } \\
\text { health } \\
\text { or medi- } \\
\text { cal con- } \\
\text { ditions } \\
\text { investi- } \\
\text { gated }\end{array}$ & $\begin{array}{l}\text { Sam- } \\
\text { pling } \\
\text { method }\end{array}$ & \multicolumn{3}{|c|}{ Sample size } & $\begin{array}{l}\text { Partici- } \\
\text { pant attri- } \\
\text { tion }\end{array}$ & $\begin{array}{l}\text { Re- } \\
\text { search } \\
\text { methods } \\
\text { or data } \\
\text { collec- } \\
\text { tion } \\
\text { tools } \\
\text { Loneli- } \\
\text { ness } \\
\text { scale } \\
\text { used }\end{array}$ \\
\hline $\begin{array}{l}\text { Lars- } \\
\text { son et } \\
\text { al } \\
(2016) \\
{[50] \text {, }} \\
\text { Swe- } \\
\text { den }\end{array}$ & High & $\begin{array}{l}\text { Random- } \\
\text { ized, } \\
\text { crossover } \\
\text { trial }\end{array}$ & $\begin{array}{l}\text { Living } \\
\text { in ordi- } \\
\text { nary } \\
\text { housing } \\
\text { without } \\
\text { any } \\
\text { home } \\
\text { care ser- } \\
\text { vices }\end{array}$ & $\begin{array}{l}\text { Range: } \\
61-89, \\
\text { mean } \\
71.2 ; \\
\text { group } 1 \\
\text { (inter- } \\
\text { vention } \\
\text { or con- } \\
\text { trol } \\
\text { group): } \\
\text { range } \\
66-89, \\
\text { mean } \\
73.4 ; \\
\text { group } 2 \\
\text { (control } \\
\text { or inter- } \\
\text { vention } \\
\text { group): } \\
\text { range } \\
61-76, \\
\text { mean } \\
69.0\end{array}$ & $\begin{array}{l}\text { Male=6; fe- } \\
\text { male=24, } \\
\text { ( } 3 \text { males } \\
\text { and } 12 \text { fe- } \\
\text { males each } \\
\text { in group } 1 \\
\text { [interven- } \\
\text { tion or con- } \\
\text { trol group] } \\
\text { and group } \\
2 \text { [control } \\
\text { or interven- } \\
\text { tion } \\
\text { group]) }\end{array}$ & $\begin{array}{l}\text { Not report- } \\
\text { ed (proba- } \\
\text { bly all } \\
\text { Swedes) }\end{array}$ & $\begin{array}{l}\text { Loneli- } \\
\text { ness }\end{array}$ & $\begin{array}{l}\text { Ran- } \\
\text { dom- } \\
\text { ized } \\
\text { (after } \\
\text { recruit- } \\
\text { ment) }\end{array}$ & 30 & $\begin{array}{l}15 \\
\text { base- } \\
\text { line, } \\
14 \text { fol- } \\
\text { low- } \\
\text { up }\end{array}$ & $\begin{array}{l}15 \\
\text { base- } \\
\text { line, } \\
14 \text { fol- } \\
\text { low- } \\
\text { up }\end{array}$ & $\begin{array}{l}2 \text { ( } 1 \text { par- } \\
\text { ticipant } \\
\text { each from } \\
\text { interven- } \\
\text { tion and } \\
\text { control } \\
\text { groups); } \\
\text { attrition } \\
\text { rate=6.7\% }\end{array}$ & $\begin{array}{l}\text { UCLA } \\
\text { loneli- } \\
\text { ness } \\
\text { scale } \\
\text { [51], the } \\
\text { Swedish } \\
\text { version } \\
(20 \\
\text { items, } \\
\text { score } \\
\text { range } \\
20-80) \\
\text { [52] }\end{array}$ \\
\hline $\begin{array}{l}\text { Czaja } \\
\text { et al } \\
(2018) \\
{[45],} \\
\text { United } \\
\text { States }\end{array}$ & High & $\begin{array}{l}\text { Multisite } \\
\text { random- } \\
\text { ized con- } \\
\text { trolled tri- } \\
\text { al }\end{array}$ & $\begin{array}{l}\text { Living } \\
\text { in inde- } \\
\text { pendent } \\
\text { housing } \\
\text { in the } \\
\text { commu- } \\
\text { nity }\end{array}$ & $\begin{array}{l}\text { Base- } \\
\text { line: to- } \\
\text { tal sam- } \\
\text { ple } \\
\text { mean } \\
76.15 \\
\text { (SD } \\
7.4) \text {, } \\
\text { range: } \\
65-98 ; \\
\text { interven- } \\
\text { tion } \\
\text { (PRISM } \\
\text { System) } \\
\text { group: } \\
\text { mean } \\
76.9 \\
\text { (SD } \\
7.3) ; \\
\text { control } \\
\text { (Binder }{ }^{\mathrm{e}} \text { ) } \\
\text { group: } \\
\text { mean } \\
75.3 \\
\text { (SD } \\
7.4)\end{array}$ & $\begin{array}{l}\text { Baseline: } \\
\text { fe- } \\
\text { male=78\% } \\
\text { (number } \\
\text { not report- } \\
\text { ed), } \\
\text { male=22\% } \\
\text { (number } \\
\text { not report- } \\
\text { ed); } \\
\text { PRISM or } \\
\text { interven- } \\
\text { tion group: } \\
\text { female } \\
79.3 \% \\
\text { (n=119); } \\
\text { Binder } \\
\text { (control) } \\
\text { group: fe- } \\
\text { male } \\
76.7 \% \\
\text { (n=115) }\end{array}$ & $\begin{array}{l}\text { Baseline: } \\
\text { White=54\% } \\
\text { and non- } \\
\text { White=46\%; } \\
\text { PRISM or } \\
\text { interven- } \\
\text { tion group: } \\
\text { non-White } \\
\text { or Hispan- } \\
\text { ic=8\% } \\
\text { (n=12); } \\
\text { Binder } \\
\text { group: } \\
\text { non-White } \\
\text { or Hispan- } \\
\text { ic=10\% } \\
\text { (n=15) }\end{array}$ & $\begin{array}{l}\text { Social } \\
\text { isola- } \\
\text { tion, so- } \\
\text { cial sup- } \\
\text { port, } \\
\text { loneli- } \\
\text { ness, } \\
\text { and } \\
\text { well-be- } \\
\text { ing }\end{array}$ & $\begin{array}{l}\text { Ran- } \\
\text { dom- } \\
\text { ized }\end{array}$ & $\begin{array}{l}300 \\
\text { (150 in } \\
\text { each in- } \\
\text { terven- } \\
\text { tion } \\
\text { [PRISM] } \\
\text { group } \\
\text { and con- } \\
\text { trol } \\
\text { [Binder] } \\
\text { group) }\end{array}$ & $\begin{array}{l}150 \\
\text { base- } \\
\text { line; } \\
134 \\
\text { fol- } \\
\text { low- } \\
\text { up }\end{array}$ & $\begin{array}{l}150 \\
\text { base- } \\
\text { line; } \\
118 \\
\text { fol- } \\
\text { low- } \\
\text { up }\end{array}$ & $\begin{array}{l}56 \text { (45 at } \\
6 \text { months } \\
\text { and } 11 \text { at } \\
12-\text { month } \\
\text { follow- } \\
\text { up); attri- } \\
\text { tion } \\
\text { rate=18.7\% }\end{array}$ & $\begin{array}{l}\text { UCLA } \\
\text { loneli- } \\
\text { ness } \\
\text { scale } \\
\text { (score } \\
\text { range } \\
20-80 \text { ) } \\
{[51]}\end{array}$ \\
\hline
\end{tabular}




\begin{tabular}{|c|c|c|c|c|c|c|c|c|c|c|c|c|c|}
\hline \multirow{2}{*}{$\begin{array}{l}\text { Study, } \\
\text { coun- } \\
\text { try }\end{array}$} & \multirow{2}{*}{$\begin{array}{l}\text { Quality } \\
\text { of evi- } \\
\text { dence }^{\mathrm{a}} \\
\text { (review- } \\
\text { ers' as- } \\
\text { sess- } \\
\text { ment) }\end{array}$} & \multirow{2}{*}{$\begin{array}{l}\text { Research } \\
\text { design }\end{array}$} & \multirow[t]{2}{*}{ Settings } & \multicolumn{3}{|c|}{ Participants } & \multirow{2}{*}{$\begin{array}{l}\text { Main } \\
\text { health } \\
\text { or medi- } \\
\text { cal con- } \\
\text { ditions } \\
\text { investi- } \\
\text { gated }\end{array}$} & \multirow{2}{*}{$\begin{array}{l}\text { Sam- } \\
\text { pling } \\
\text { method }\end{array}$} & \multicolumn{3}{|c|}{ Sample size } & \multirow{2}{*}{$\begin{array}{l}\text { Partici- } \\
\text { pant attri- } \\
\text { tion }\end{array}$} & \multirow{2}{*}{$\begin{array}{l}\text { Re- } \\
\text { search } \\
\text { methods } \\
\text { or data } \\
\text { collec- } \\
\text { tion } \\
\text { tools } \\
\text { Loneli- } \\
\text { ness } \\
\text { scale } \\
\text { used }\end{array}$} \\
\hline & & & & $\begin{array}{l}\text { Age } \\
\text { (years) }\end{array}$ & Gender & Ethnicity & & & Total & $\begin{array}{l}\text { Inter- } \\
\text { ven- } \\
\text { tion } \\
\text { group }\end{array}$ & $\begin{array}{l}\text { Con- } \\
\text { trol } \\
\text { group }\end{array}$ & & \\
\hline $\begin{array}{l}\text { Mor- } \\
\text { ton et } \\
\text { al } \\
(2018) \\
{[53] \text {, }} \\
\text { United } \\
\text { King- } \\
\text { dom }\end{array}$ & High & $\begin{array}{l}2 \text { (condi- } \\
\text { tion: } \\
\text { training, } \\
\text { con- } \\
\text { trol)×2 } \\
\text { (popula- } \\
\text { tion: } \\
\text { domicil- } \\
\text { iary, resi- } \\
\text { den- } \\
\text { tial) } \times 2 \\
\text { (time: } \\
\text { baseline, } \\
\text { follow- } \\
\text { up) de- } \\
\text { sign }\end{array}$ & $\begin{array}{l}\text { Receiv- } \\
\text { ing care } \\
\text { in own } \\
\text { home or } \\
\text { support- } \\
\text { ed hous- } \\
\text { ing in } \\
\text { the com- } \\
\text { munity } \\
\text { (domicil- } \\
\text { iary } \\
\text { care) or } \\
\text { residen- } \\
\text { tial care } \\
\text { in care } \\
\text { homes }\end{array}$ & $\begin{array}{l}\text { Female: } \\
\text { mean } \\
80.71 \\
(\mathrm{SD} \\
8.77) ; \\
\text { male: } \\
\text { data not } \\
\text { reported }\end{array}$ & $\begin{array}{l}\text { Follow-up: } \\
\text { total }=76 ; \\
\text { female }=50 \\
\text { male }=26\end{array}$ & $\begin{array}{l}\text { Not report- } \\
\text { ed }\end{array}$ & $\begin{array}{l}\text { Well-be- } \\
\text { ing and } \\
\text { social } \\
\text { support }\end{array}$ & $\begin{array}{l}\text { Ran- } \\
\text { dom- } \\
\text { ized }\end{array}$ & $\begin{array}{l}97 \text { base- } \\
\text { line; } 76 \\
\text { follow- } \\
\text { up }\end{array}$ & $\begin{array}{l}53 \\
\text { base- } \\
\text { line; } \\
44 \text { fol- } \\
\text { low- } \\
\text { up }\end{array}$ & $\begin{array}{l}44 \\
\text { base- } \\
\text { line; } \\
32 \text { fol- } \\
\text { low- } \\
\text { up }\end{array}$ & $\begin{array}{l}21 \text { (9 ex- } \\
\text { perimen- } \\
\text { tal group; } \\
12 \text { con- } \\
\text { trol } \\
\text { group); } \\
\text { attrition } \\
\text { rate=21.6\% }\end{array}$ & $\begin{array}{l}\text { UCLA } \\
\text { loneli- } \\
\text { ness } \\
\text { scale } \\
\text { (score } \\
\text { range } \\
20-80 \text { ) } \\
{[51]}\end{array}$ \\
\hline $\begin{array}{l}\text { Jarvis } \\
\text { et al } \\
(2019) \\
{[54],} \\
\text { South } \\
\text { Africa }\end{array}$ & High & $\begin{array}{l}\text { Random- } \\
\text { ized con- } \\
\text { trol study }\end{array}$ & $\begin{array}{l}\text { Inner- } \\
\text { city resi- } \\
\text { dential; } \\
\text { NGO }^{f} \\
\text { care fa- } \\
\text { cilities } \\
\text { for re- } \\
\text { source- } \\
\text { restrict- } \\
\text { ed older } \\
\text { people } \\
\text { (aged } \\
\geq 60 \\
\text { years) }\end{array}$ & $\begin{array}{l}\text { Mean } \\
74.93 \\
(\mathrm{SD} \\
6.41) ; \\
\text { range } \\
61-87\end{array}$ & $\begin{array}{l}\text { Baseline: } \\
\text { male=6 } \\
(18.8 \%), \\
\text { female=26 } \\
(81.2 \%)\end{array}$ & $\begin{array}{l}\text { Mostly } \\
\text { Asian (of } \\
\text { Indian ori- } \\
\text { gin), num- } \\
\text { bers not re- } \\
\text { ported }\end{array}$ & $\begin{array}{l}\text { Mal- } \\
\text { adaptive } \\
\text { cogni- } \\
\text { tions } \\
\text { and } \\
\text { loneli- } \\
\text { ness }\end{array}$ & $\begin{array}{l}\text { Ran- } \\
\text { dom- } \\
\text { ized }\end{array}$ & $\begin{array}{l}\text { Base- } \\
\text { line=32 } \\
\text { (inter- } \\
\text { vention } \\
\text { group=15, } \\
\text { control } \\
\text { group=17), } \\
\text { final=29 } \\
\text { (inter- } \\
\text { vention } \\
\text { group=13, } \\
\text { control } \\
\text { group=16) }\end{array}$ & $\begin{array}{l}15 \\
\text { base- } \\
\text { line; } \\
13 \text { fol- } \\
\text { low- } \\
\text { up }\end{array}$ & $\begin{array}{l}17 \\
\text { base- } \\
\text { line; } \\
16 \text { fol- } \\
\text { low- } \\
\text { up }\end{array}$ & $\begin{array}{l}3 \text { ( } 2 \text { inter- } \\
\text { vention } \\
\text { group, } 1 \\
\text { control } \\
\text { group); } \\
\text { attrition } \\
\text { rate }=15.6 \%\end{array}$ & $\begin{array}{l}\text { De } \\
\text { Jong- } \\
\text { Gierveld } \\
\text { loneli- } \\
\text { ness } \\
\text { scale } \\
\text { (score } \\
\text { range 0- } \\
\text { 11) [49] }\end{array}$ \\
\hline
\end{tabular}

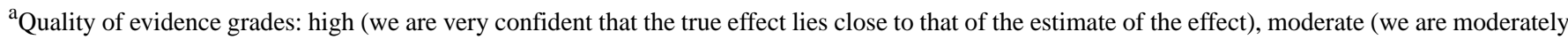
confident in the effect estimate: the true effect is likely to be close to the estimate of the effect, but there is a possibility that it is substantially different), low (our confidence in the effect estimate is limited: the true effect may be substantially different from the estimate of the effect), and very low (we have very little confidence in the effect estimate; the true effect is likely to be substantially different from the estimate of effect).

bRCT: nonrandomized clinical trial.

${ }^{\mathrm{c}}$ UCLA: University of California, Los Angeles.

${ }^{\mathrm{d}}$ PRISM: Personal Reminder Information and Social Management.

${ }^{\mathrm{e}}$ Binder refers to a group of participants who received a notebook with printed content similar to the Personal Reminder Information and Social Management System.

${ }^{\mathrm{f}} \mathrm{NGO}$ : nongovernmental organization. 
Table 2. Interventions, outcomes, measurements, results, and conclusions of included studies.

\begin{tabular}{|c|c|c|c|c|c|c|c|c|c|c|c|}
\hline \multirow[t]{2}{*}{ Study } & \multirow{2}{*}{$\begin{array}{l}\text { Interven- } \\
\text { tions }\end{array}$} & \multirow{2}{*}{$\begin{array}{l}\text { Compara- } \\
\text { tors }\end{array}$} & \multirow{2}{*}{$\begin{array}{l}\text { Inter- } \\
\text { ven- } \\
\text { tion } \\
\text { dura- } \\
\text { tion }\end{array}$} & \multirow{2}{*}{$\begin{array}{l}\text { Fol- } \\
\text { low- } \\
\text { up du- } \\
\text { ration }\end{array}$} & \multicolumn{5}{|c|}{ Outcomes: loneliness scores by measurement stages, mean (SD) } & \multirow{2}{*}{$\begin{array}{l}\text { Results or find- } \\
\text { ings }\end{array}$} & \multirow{2}{*}{$\begin{array}{l}\text { Conclusion } \\
\text { by the au- } \\
\text { thors of the } \\
\text { study }\end{array}$} \\
\hline & & & & & Baseline & 3 months & 4 months & 6 months & $\begin{array}{l}12 \\
\text { months }\end{array}$ & & \\
\hline $\begin{array}{l}\text { Tsai } \\
\text { et al } \\
(2010) \\
{[46]}\end{array}$ & $\begin{array}{l}\text { Videocon- } \\
\text { ferencing } \\
\text { (using ei- } \\
\text { ther } \\
\text { MSN }^{\mathrm{a}} \\
\text { messen- } \\
\text { ger or } \\
\text { Skype) }\end{array}$ & $\begin{array}{l}\text { Regular } \\
\text { care }\end{array}$ & $\begin{array}{l}3 \\
\text { months }\end{array}$ & $\begin{array}{l}3 \\
\text { months }\end{array}$ & $\begin{array}{l}\text { Interven- } \\
\text { tion } \\
\text { group=50.58 } \\
(\mathrm{SD} \\
11.16) ; \\
\text { control } \\
\text { group=46.55 } \\
(\mathrm{SD} 9.07)\end{array}$ & $\begin{array}{l}\text { Interven- } \\
\text { tion } \\
\text { group=47.33 } \\
(\mathrm{SD} \\
13.50) \text {; } \\
\text { control } \\
\text { group=46.68 } \\
(\mathrm{SD} 9.08)\end{array}$ & $\begin{array}{l}\text { Not mea- } \\
\text { sured }\end{array}$ & $\begin{array}{l}\text { Not mea- } \\
\text { sured }\end{array}$ & $\begin{array}{l}\text { Not mea- } \\
\text { sured }\end{array}$ & $\begin{array}{l}\text { Loneliness: inter- } \\
\text { vention group } \\
\text { mean: baseline } \\
50.58 \text { (SD } 11.16 \text { ), } \\
1 \text { week } 49.75 \\
\text { (SD 11.79), and } \\
3 \text { months } 47.33 \\
\text { (SD 13.50); con- } \\
\text { trol group mean: } \\
\text { baseline } 46.55 \\
\text { (SD 9.07), } 1 \\
\text { week } 47.06 \text { (SD } \\
8.75), \text { and } 3 \\
\text { months } 46.68 \\
\text { (SD 9.08); differ- } \\
\text { ences between } \\
\text { groups were } \\
\text { compared at } 3 \\
\text { points (baseline, } \\
1 \text { week, and } 3 \\
\text { months) using } \\
\text { multiple linear } \\
\text { regression of the } \\
\text { generalized esti- } \\
\text { mating equations. } \\
\text { Unadjusted or } \\
\text { fixed effect size } \\
\text { of effectiveness } \\
\text { of videoconfer- } \\
\text { encing interven- } \\
\text { tion (videoconfer- } \\
\text { ence vs control): } \\
\text { at } 1 \text { week was } \\
\beta=-1.21, \text { SE } \\
0.50, \chi^{2}=5.9, \\
P=.02 \text { and at } 3 \\
\text { months } \beta=-2.84 \text {, } 1.28, \chi^{2}=4.9 \text {, }\end{array}$ & $\begin{array}{l}\text { Videoconfer- } \\
\text { encing allevi- } \\
\text { ates depres- } \\
\text { sive symp- } \\
\text { toms and } \\
\text { loneliness in } \\
\text { older resi- } \\
\text { dents in } \\
\text { nursing } \\
\text { homes }\end{array}$ \\
\hline $\begin{array}{l}\text { van } \\
\text { der } \\
\text { Hei- } \\
\text { de et } \\
\text { al } \\
(2012) \\
{[48]}\end{array}$ & $\begin{array}{l}\text { CareTV } \\
\text { including } \\
\text { Caret du- } \\
\text { plex } \\
\text { video or } \\
\text { voice net- } \\
\text { work }\end{array}$ & $\begin{array}{l}\text { No control } \\
\text { group and } \\
\text { no com- } \\
\text { parator }\end{array}$ & $\begin{array}{l}12 \\
\text { months }\end{array}$ & $\begin{array}{l}12 \\
\text { months }\end{array}$ & $\begin{array}{l}\text { Interven- } \\
\text { tion } \\
\text { group=5.97 } \\
\text { (SD 2.77); } \\
\text { no control } \\
\text { group }\end{array}$ & $\begin{array}{l}\text { Not mea- } \\
\text { sured }\end{array}$ & $\begin{array}{l}\text { Not mea- } \\
\text { sured }\end{array}$ & $\begin{array}{l}\text { Not mea- } \\
\text { sured }\end{array}$ & $\begin{array}{l}\text { Interven- } \\
\text { tion } \\
\text { group=4.02 } \\
(\mathrm{SD} \\
3.91) \text {; no } \\
\text { control } \\
\text { group }\end{array}$ & $\begin{array}{l}\text { Group-level total } \\
\text { loneliness: inclu- } \\
\text { sion stage: mean } \\
5.97 \text { (SD 2.77), } \\
\text { end of study: } \\
\text { mean } 4.02 \text { (SD } \\
3.91 \text { ), } P=.001 \text {; in- } \\
\text { dividual-level to- } \\
\text { tal loneliness: to- } \\
\text { tal loneliness de- } \\
\text { creased in } 54 \text { out } \\
\text { of } 85 \text { participants } \\
\text { (equally lonely } \\
11 \text {, more lonely } \\
20 \text {, and less lone- } \\
\text { ly } 54 \text { individual } \\
\text { participants) }\end{array}$ & $\begin{array}{l}\text { CareTV inter- } \\
\text { vention de- } \\
\text { creased the } \\
\text { feeling of } \\
\text { loneliness in } \\
\text { the partici- } \\
\text { pants; howev- } \\
\text { er, partici- } \\
\text { pants were } \\
\text { feeling mod- } \\
\text { erate loneli- } \\
\text { ness at the } \\
\text { end of the } \\
\text { study }\end{array}$ \\
\hline
\end{tabular}




\begin{tabular}{|c|c|c|c|c|c|c|c|c|c|c|c|}
\hline \multirow[t]{2}{*}{ Study } & \multirow{2}{*}{$\begin{array}{l}\text { Interven- } \\
\text { tions }\end{array}$} & \multirow{2}{*}{$\begin{array}{l}\text { Compara- } \\
\text { tors }\end{array}$} & \multirow{2}{*}{$\begin{array}{l}\text { Inter- } \\
\text { ven- } \\
\text { tion } \\
\text { dura- } \\
\text { tion }\end{array}$} & \multirow{2}{*}{$\begin{array}{l}\text { Fol- } \\
\text { low- } \\
\text { up du- } \\
\text { ration }\end{array}$} & \multicolumn{5}{|c|}{ Outcomes: loneliness scores by measurement stages, mean (SD) } & \multirow{2}{*}{$\begin{array}{l}\text { Results or find- } \\
\text { ings }\end{array}$} & \multirow{2}{*}{$\begin{array}{l}\text { Conclusion } \\
\text { by the au- } \\
\text { thors of the } \\
\text { study }\end{array}$} \\
\hline & & & & & Baseline & 3 months & 4 months & 6 months & $\begin{array}{l}12 \\
\text { months }\end{array}$ & & \\
\hline $\begin{array}{l}\text { Lars- } \\
\text { son } \\
\text { et al } \\
(2016) \\
{[50]}\end{array}$ & $\begin{array}{l}\text { SIBAs }{ }^{b}, \\
\text { that is, so- } \\
\text { cial activi- } \\
\text { ties via } \\
\text { social } \\
\text { websites }\end{array}$ & $\begin{array}{l}\text { No com- } \\
\text { parator in- } \\
\text { tervention } \\
\text { reported }\end{array}$ & $\begin{array}{l}3 \\
\text { months }\end{array}$ & $\begin{array}{l}34 \\
\text { weeks } \\
\text { (expo- } \\
\text { sure } \\
\text { for } 3 \\
\text { months } \\
\text { to } \\
\text { each } \\
\text { group) }\end{array}$ & $\begin{array}{l}\text { Group } 1 \\
\left(\mathrm{I} / \mathrm{C}^{\mathrm{c}}\right. \\
\text { group }=45.53 \\
(\mathrm{SD} 7.41) ; \\
\text { group } 2 \\
\left(\mathrm{C} / \mathrm{I}^{\mathrm{d}}\right. \\
\text { group})=43.93 \\
(\mathrm{SD} 8.61)\end{array}$ & $\begin{array}{l}\text { Group } 1 \\
\text { (I/C } \\
\text { group)=4243 } \\
(\mathrm{SD} \\
7.44) ; \\
\text { group } 2 \\
(\mathrm{C} / \mathrm{I} \\
\text { group) }=41.93 \\
(\mathrm{SD} 8.82)\end{array}$ & $\begin{array}{l}\text { Not mea- } \\
\text { sured }\end{array}$ & $\begin{array}{l}3 \text { months } \\
\text { after cross } \\
\text { over: group } \\
1 \text { (I/C } \\
\text { group, no } \\
\text { interven- } \\
\text { tion)=42.0 } \\
\text { (SD 7.34); } \\
\text { group } 2 \\
\text { (C/I group, } \\
\text { interven- } \\
\text { tion intro- } \\
\text { duced)=39.50 } \\
\text { (SD 10.42) }\end{array}$ & $\begin{array}{l}\text { Not mea- } \\
\text { sured }\end{array}$ & $\begin{array}{l}\text { Percentage } \\
\text { change between } \\
\text { time } 2 \text { and time } \\
\text { 1: group 1: mean } \\
\text { score } 0.07 \% \text { (SD } \\
0.07), P=.003 ; \\
\text { group } 2: \text { mean } \\
\text { score: } 0.05 \% \text { (SD } \\
0.09), P=.049 ; \\
\text { percentage } \\
\text { change between } \\
\text { time } 3 \text { and time } \\
1: \text { group } 1: \text { mean } \\
\text { score } 0.08 \% \text { (SD } \\
0.08) \text {; group } 2: \\
\text { mean score } \\
0.09 \% \text { (SD } 0.13) ; \\
\text { comparison of } \\
\text { pre and postinter- } \\
\text { vention scores: } \\
\text { group } 1, P=.003 \\
\text { and group } 2, \\
P=.049\end{array}$ & $\begin{array}{l}\text { SIBA inter- } \\
\text { ventions } \\
\text { have the po- } \\
\text { tential to re- } \\
\text { duce experi- } \\
\text { ences of } \\
\text { loneliness in } \\
\text { socially vul- } \\
\text { nerable older } \\
\text { adults. }\end{array}$ \\
\hline $\begin{array}{l}\text { Cza- } \\
\text { ja et } \\
\text { al } \\
(2018) \\
{[45]}\end{array}$ & $\begin{array}{l}\text { PRISM }^{\mathrm{e}} \\
\text { system }\end{array}$ & $\begin{array}{l}\text { A note- } \\
\text { book with } \\
\text { printed } \\
\text { content } \\
\text { similar to } \\
\text { that within } \\
\text { the PRISM } \\
\text { (interven- } \\
\text { tion) } \\
\text { group: in- } \\
\text { cluded a } \\
\text { Lenovo Mi- } \\
\text { ni Desktop } \\
\text { PC with a } \\
\text { keyboard, } \\
\text { mouse (or } \\
\text { trackball } \\
\text { for those } \\
\text { who were } \\
\text { unable to } \\
\text { control a } \\
\text { mouse), a } \\
19^{\prime \prime} \text { LCD } \\
\text { monitor, } \\
\text { the PRISM } \\
\text { software } \\
\text { app, a } \\
\text { printer, and } \\
\text { internet }\end{array}$ & $\begin{array}{l}12 \\
\text { months }\end{array}$ & $\begin{array}{l}12 \\
\text { months }\end{array}$ & $\begin{array}{l}\text { Interven- } \\
\text { tion } \\
(\text { PRISM) } \\
\text { group=39.8 } \\
(\text { SD 9.7); } \\
\text { control } \\
\left(\text { Binder }{ }^{\mathrm{g}}\right) \\
\text { group=40.2 } \\
(\mathrm{SD} 10.3)\end{array}$ & $\begin{array}{l}\text { Not mea- } \\
\text { sured }\end{array}$ & $\begin{array}{l}\text { Not mea- } \\
\text { sured }\end{array}$ & $\begin{array}{l}\text { Interven- } \\
\text { tion } \\
\text { (PRISM) } \\
\text { group=37.8 } \\
\text { (SD 9.54); } \\
\text { control } \\
\text { (Binder) } \\
\text { group=40 } \\
\text { (SD 10.62) }\end{array}$ & $\begin{array}{l}\text { Interven- } \\
\text { tion } \\
(\mathrm{PRISM}) \\
\text { group=36.9 } \\
(\mathrm{SD} \\
9.16) ; \\
\text { control } \\
(\text { Binder) } \\
\text { group=38.43 } \\
(\mathrm{SD} 9.37)\end{array}$ & $\begin{array}{l}\text { Baseline: loneli- } \\
\text { ness PRISM } \\
\text { group: mean } \\
\text { score } 39.8 \text { (SD } \\
\text { 9.7); Binder } \\
\text { group: mean } \\
\text { score 40.2 (SD } \\
\text { 10.3), follow-up } \\
\text { at } 6 \text { months: } \\
\text { PRISM group } \\
\text { 37.8, Binder } \\
\text { group 39.6; fol- } \\
\text { low-up at 12 } \\
\text { months: PRISM } \\
\text { group 36.9, } \\
\text { Binder group } \\
38.3\end{array}$ & $\begin{array}{l}\text { Technology- } \\
\text { based apps } \\
\text { such as the } \\
\text { PRISM sys- } \\
\text { tem may en- } \\
\text { hance social } \\
\text { connectivity } \\
\text { and reduce } \\
\text { loneliness } \\
\text { among older } \\
\text { adults. }\end{array}$ \\
\hline
\end{tabular}




\begin{tabular}{|c|c|c|c|c|c|c|c|c|c|c|c|}
\hline \multirow[t]{2}{*}{ Study } & \multirow{2}{*}{$\begin{array}{l}\text { Interven- } \\
\text { tions }\end{array}$} & \multirow{2}{*}{$\begin{array}{l}\text { Compara- } \\
\text { tors }\end{array}$} & \multirow{2}{*}{$\begin{array}{l}\text { Inter- } \\
\text { ven- } \\
\text { tion } \\
\text { dura- } \\
\text { tion }\end{array}$} & \multirow{2}{*}{$\begin{array}{l}\text { Fol- } \\
\text { low- } \\
\text { up du- } \\
\text { ration }\end{array}$} & \multicolumn{5}{|c|}{ Outcomes: loneliness scores by measurement stages, mean (SD) } & \multirow{2}{*}{$\begin{array}{l}\text { Results or find- } \\
\text { ings }\end{array}$} & \multirow{2}{*}{$\begin{array}{l}\text { Conclusion } \\
\text { by the au- } \\
\text { thors of the } \\
\text { study }\end{array}$} \\
\hline & & & & & Baseline & 3 months & 4 months & 6 months & $\begin{array}{l}12 \\
\text { months }\end{array}$ & & \\
\hline $\begin{array}{l}\text { Mor- } \\
\text { ton } \\
\text { et al } \\
(2018) \\
{[53]}\end{array}$ & $\begin{array}{l}\text { EasyPC-a } \\
\text { cus- } \\
\text { tomized } \\
\text { computer } \\
\text { platform } \\
\text { with a } \\
\text { simpli- } \\
\text { fied } \\
\text { touch- } \\
\text { screen in- } \\
\text { terface }\end{array}$ & $\begin{array}{l}\text { Care as } \\
\text { usual plus } \\
\text { regular car- } \\
\text { er visits }\end{array}$ & $\begin{array}{l}3 \\
\text { months }\end{array}$ & $\begin{array}{l}4 \\
\text { months }\end{array}$ & $\begin{array}{l}\text { Interven- } \\
\text { tion (train- } \\
\text { ing) group } \\
\text { (total of } \\
\text { residential } \\
\text { and domi- } \\
\text { ciliary } \\
\text { groups)=1.92 } \\
\text { (SE 0.10, } \\
\text { SD 0.73); } \\
\text { control } \\
\text { group (to- } \\
\text { tal of resi- } \\
\text { dential and } \\
\text { domiciliary } \\
\text { groups)=2.08 } \\
\text { (SE 0.12, } \\
\text { SD 0.80) }\end{array}$ & $\begin{array}{l}\text { Not mea- } \\
\text { sured }\end{array}$ & $\begin{array}{l}\text { Interven- } \\
\text { tion (train- } \\
\text { ing) group } \\
\text { (total of } \\
\text { residential } \\
\text { and domi- } \\
\text { ciliary } \\
\text { groups)=1.86 } \\
\text { (SE 0.10, } \\
\text { SD 0.66); } \\
\text { control } \\
\text { group (to- } \\
\text { tal of resi- } \\
\text { dential and } \\
\text { domiciliary } \\
\text { groups)=2.12 } \\
\text { (SE 0.11, } \\
\text { SD 0.62) }\end{array}$ & $\begin{array}{l}\text { Not mea- } \\
\text { sured }\end{array}$ & $\begin{array}{l}\text { Not mea- } \\
\text { sured }\end{array}$ & 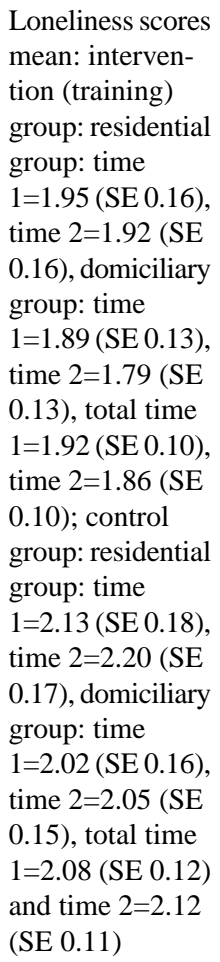 & $\begin{array}{l}\text { Internet ac- } \\
\text { cess and } \\
\text { training can } \\
\text { support the } \\
\text { self and so- } \\
\text { cial connect- } \\
\text { edness of } \\
\text { vulnerable } \\
\text { older adults } \\
\text { and con- } \\
\text { tribute posi- } \\
\text { tively to } \\
\text { well-being. }\end{array}$ \\
\hline $\begin{array}{l}\text { Jarvis } \\
\text { et al } \\
(2019) \\
{[54]}\end{array}$ & $\begin{array}{l}\text { Living In } \\
\text { Network- } \\
\text { Connect- } \\
\text { ed Com- } \\
\text { munities } \\
\text { What- } \\
\text { sApp } \\
\text { group for } \\
\text { low-inten- } \\
\text { sity cogni- } \\
\text { tive be- } \\
\text { havioral } \\
\text { therapy }\end{array}$ & $\begin{array}{l}\text { Usual care, } \\
\text { a separate } \\
\text { WhatsApp } \\
\text { group (Liv- } \\
\text { ing In Net- } \\
\text { work-Con- } \\
\text { nected } \\
\text { Communi- } \\
\text { ties 2) }\end{array}$ & $\begin{array}{l}3 \\
\text { months }\end{array}$ & $\begin{array}{l}4 \\
\text { months }\end{array}$ & $\begin{array}{l}\text { Not mea- } \\
\text { sured }\end{array}$ & $\begin{array}{l}\text { Interven- } \\
\text { tion } \\
\text { group=2.31 } \\
(\mathrm{SD} \\
1.49) ; \\
\text { control } \\
\text { group=2.47 } \\
(\mathrm{SD} 2.1)\end{array}$ & $\begin{array}{l}\text { Interven- } \\
\text { tion } \\
\text { group=1.38 } \\
(\mathrm{SD} 1.33) ; \\
\text { control } \\
\text { group=4.0 } \\
(\mathrm{SD} 1.32)\end{array}$ & $\begin{array}{l}\text { Not mea- } \\
\text { sured }\end{array}$ & $\begin{array}{l}\text { Not mea- } \\
\text { sured }\end{array}$ & $\begin{array}{l}\text { Loneliness levels: } \\
\text { total=baseline-in- } \\
\text { tervention on } \\
\text { time } 1 \text {-interven- } \\
\text { tion on time } 2 . ; \\
\chi^{2}=14.6 ; P=.001\end{array}$ & $\begin{array}{l}\text { Low-intensi- } \\
\text { ty cognitive } \\
\text { behavioral } \\
\text { therapy mo- } \\
\text { bile health } \\
\text { supported by } \\
\text { the social } \\
\text { networking } \\
\text { platform of } \\
\text { WhatsApp } \\
\text { (Living In } \\
\text { Network- } \\
\text { Connected } \\
\text { Communi- } \\
\text { ties) showed } \\
\text { significant } \\
\text { improve- } \\
\text { ments in } \\
\text { loneliness } \\
\text { and maladap- } \\
\text { tive cogni- } \\
\text { tions. }\end{array}$ \\
\hline
\end{tabular}

${ }^{\mathrm{a}}$ MSN: Microsoft Network.

${ }^{\mathrm{b}}$ SIBA: social internet-based activity.

${ }^{\mathrm{c}} \mathrm{I} / \mathrm{C}$ : intervention/control.

${ }^{\mathrm{d}} \mathrm{C} / \mathrm{I}$ : control/intervention.

${ }^{e}$ PRISM: Personal Reminder Information and Social Management.

${ }^{f}$ LCD: liquid-crystal display.

${ }^{\mathrm{g}}$ Binder refers to a group of participants who received a notebook with printed content similar to the Personal Reminder Information and Social Management System. 
SGSS and DN independently extracted data from all included studies $(n=6)$ using the data extraction template (Tables 1 and 2) and resolved discrepancies in the extracted data with discussion and agreement. Data extraction forms were compared and contrasted, thereby avoiding bias and reducing errors in the data extraction process [55]. We extracted aggregated data at the study level as much as possible with respect to the intervention, which is imperative for the reproducibility of effective interventions [56,57]. Following suggestions for reporting data once from studies with duplicate and multiple publications [55], we extracted and reported data only once [50] from a research study with multiple publications [50,58].

\section{Data Synthesis and Reporting}

We report both a narrative synthesis (narrative summary) and a statistical (quantitative) synthesis (meta-analysis) of our review, as suggested for reporting of a systematic review on effectiveness [59]. In the narrative synthesis, we have included all 6 studies and reported their characteristics, including the study design, settings, sample sizes, data collection methods, participants, interventions, comparators, outcome measurements, and study conclusions.

In the meta-analysis, we have included 5 studies and pooled extracted data on loneliness measured by continuous loneliness scales, that is, the University of California, Los Angeles (UCLA) loneliness scale [47,51] in 4 studies and the De Jong-Gierveld loneliness scale [49] in 2 studies (Table 1). Loneliness scores at baseline and follow-up were reported as the mean values and SDs in 5 studies, whereas 1 study reported mean scores with SEs. For the latter study, we calculated SDs from SEs using a formula suggested in the Cochrane guidelines [60].

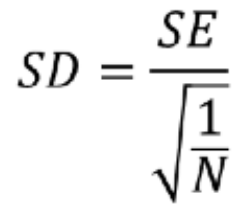

In meta-analysis, the standardized mean difference (SMD) as a summary statistic for reporting continuous data has been suggested for studies that assess the same outcome but use different scales to measure the outcome [60]. In RevMan (The Cochrane Collaboration), the SMD is the effect size known as Hedges (adjusted) g, which is akin to Cohen $d$ and includes an adjustment for small sample size bias [60]. More importantly, the generalizability of the SMD statistic is more than the mean difference statistic in a meta-analysis [61].

In our review, the main outcome, that is, loneliness, was measured using different loneliness scales, which included the UCLA loneliness scale (score range 20-80) [47,51] and the De Jong-Gierveld loneliness scale (score range 0-11) [49]. Although these 2 loneliness scales have commonalities such as self-reporting measures and focus on the functional dimension of social relationship and the degree of subjectivity covering perceived availability, adequacy, and emotions or feelings, they differ from each other in other aspects, such as the content and formulation of items or questions included in the scales [62]. In addition, the 2 measures have different number of items or questions, rating options, scoring methods, total scores, and scale versions (for details, refer to the studies by Russell [51] and Russell et al [47] for the UCLA loneliness scale and the studies by De Jong-Gierveld and Tilburg [49] and De Jong-Gierveld and Kamphuls [63] for the De Jong-Gierveld loneliness scale).

The Cochrane guidelines for systematic reviews and meta-analysis [60] suggest that different study designs should not be combined in a meta-analysis because it can increase heterogeneity, and studies with repeated measurements at different follow-up periods cannot be combined without a unit of analysis error.

We extracted data from 6 studies, which included 5 clinical trials $[45,46,50,53,54]$ and 1 pre-post study [48]. Therefore, we included similar study designs, that is, clinical trials in the meta-analysis, and conducted separate meta-analyses based on the same follow-up measurement periods in the clinical trials. Therefore, we performed a separate meta-analysis for each follow-up, that is, measurements at 3, 4, and 6 months after the intervention. In addition, we ran meta-analyses when there were at least two or more studies for the same outcome or the same follow-up period [64]. Therefore, we did not conduct a meta-analysis for follow-up measurements at 12 months reported in 2 studies because they involved different study designs, that is, a randomized controlled trial (RCT) with intervention and control groups [45] and a pre- and postintervention study with only intervention group [48]. This was done to avoid an increase in the heterogeneity [60] and overestimation of the effect of intervention in the absence of a control group [65] in the preand postintervention study [48]. We did not perform a meta-analysis for the pre-post study [48] because meta-analysis cannot be performed with only 1 study [60].

We calculated the SMDs from the extracted data, that is, loneliness mean scores with SD and sample sizes in the intervention and control groups at follow-up measurements at 3 months and beyond. For conducting meta-analysis, we used the Cochrane Review Manager (RevMan) software, version 5.3.5 [66]. In the meta-analysis, we used the random effects model as the statistical model because we hypothesized that the true effect sizes between studies would vary $[67,68]$ due to differences in the methodological and clinical characteristics between studies [69], such as differences in the sample sizes, participant numbers and characteristics, intervention types and durations, and follow-up measurement times. We did not conduct sensitivity analyses because of the small number of studies in the meta-analyses at each follow-up point [64].

\section{Assessment of Research Quality, Bias, and Heterogeneity}

We assessed the quality of research by applying the GRADE (Grading of Recommendations Assessment, Development and Evaluation) approach [70].

We assessed the risk of bias by focusing on 5 domains: the evaluation of sequence generation, allocation concealment, blinding (outcome assessors), incomplete data, selective outcome reporting, and assessing other biases using the Cochrane guidelines [60]. In a meta-analysis, publication bias can be assessed with a graphical method using funnel plots 
[60,71] and statistical methods such as the Egger test [60]; however, both methods require at least 10 studies in the meta-analysis [60]. When the number of studies is small, the Egger test has low power and fails to differentiate chance from real asymmetry [60]. Similarly, assessing publication bias using funnel plots with fewer studies would be of very limited usefulness because it would be difficult to spot the publication bias. As we had a maximum of 3 studies in a meta-analysis, we could not check the publication bias with either method.

We checked heterogeneity, that is, variation in study outcomes or intervention effect sizes between studies, by the Cochran Q test with a significance level of $\rho<0.10[72,73]$ because of the low power of the test in a meta-analysis with very few studies or studies with small sample sizes [74]. We calculated $\mathrm{I}^{2}$ statistics to determine the magnitude of heterogeneity (ie, the proportion of variance in the true effect sizes) between studies [28]. We considered $\mathrm{I}^{2}$ values of $25 \%, 50 \%$, and $75 \%$ as low, moderate, and high heterogeneity between studies, respectively [75].

\section{Summary Measures}

We report the findings of meta-analyses using SMDs with $95 \%$ CIs as a statistical summary, with the forest plots [60].

\section{Results}

\section{Narrative Synthesis}

Findings about the characteristics of the studies, including the study designs, settings, participants, interventions, comparators, sample sizes, participant attrition, and data collection methods or tools used, are presented in Table 1. The interventions, comparators, follow-up durations, outcomes or measurement scores, results, and conclusions of the included studies are given in Table 2.

\section{Study Selection}

Searches of PubMed, MEDLINE, CINAHL, Embase, and Web of Science generated a total of 4939 articles (Figure 1), of which 6 studies met the predefined eligibility criteria. All 6 studies were included in the narrative synthesis, 5 clinical trials with the intervention and control groups were included in the meta-analysis, and only 1 study with a pre-post design involving only the intervention group was excluded from the meta-analysis.

\section{Study Participants}

The total number of participants enrolled in all 6 included studies was 646 (mean 108, SD 102; median 77, IQR 32-130). Studies varied in total sample sizes (mean 108, SD 102; range 30-300), and the sample sizes of the intervention and control groups also varied at both the baseline and follow-up measurements across the studies (Table 1). The attrition rate also varied between studies (range 7\%-35\%; mean 19\%, SD $10 \%)$.

Participants' average age was between 73 and 78 years (SD 6-11). Total enrolled participants included $66.1 \%(427 / 646)$ women and $23.8 \%$ (154/646) men, whereas for $10.1 \%(65 / 646)$ of participants, no information about their gender was available.
Studies varied in the proportion of male and female participants (female: mean $66 \%$, SD 16\%; range $46 \%-81 \%$; male: mean $25 \%$, SD 9\%; range 19\%-42\%). Only 2 studies reported on participants' ethnicity-White (54\%) and non-Whites (46\%) in the US study [45] and mostly Asian Indians (no numbers reported) in the South African study [54].

\section{Study Characteristics}

A total of 4 studies were RCTs [45,50,53,54], 1 study was a nonrandomized clinical trial [46], and 1 was a pre- and posttest (before and after) study with intervention group only (no control group) [48] (Table 1).

\section{Study Settings}

A total of 4 studies were conducted in developed countries, namely, the Netherlands [48], the United Kingdom [53], the United States [45], and Sweden [50]. Two studies were undertaken in developing countries, namely, Taiwan [46] and South Africa [54].

The settings included living in independent housing in the community [45]; living in ordinary housing without any home care services [50]; receiving care in their own home or supported housing in the community (domiciliary care), or receiving care in residential care homes [53], residential care facilities for older people [54], nursing homes [46], and older home care [48].

Participants were selected by random sampling in $66.7 \%(4 / 6)$ of studies $[45,50,53,54]$, whereas the other $33.3 \%(2 / 6)$ studies used purposive [46] and convenience [48] sampling each.

\section{Digital Technology Interventions}

DTIs included social internet-based activities, that is, social activities via social websites [50], videoconferencing [46], customized computer platforms with simplified touch-screen interfaces [53], personal reminder information and social management systems [45], WhatsApp groups [54], and video or voice networks [48].

\section{Duration of the Intervention and Measurement of the Main Outcome Measure}

The duration of the intervention was 3 months in 4 studies $[46,50,53,54]$ and 12 months in 2 studies $[45,48]$. The main outcome measure, that is, loneliness, was measured at the baseline and multiple follow-up times, which included 3 months in 3 studies [46,50,54], 4 months in 2 studies [53,54], 6 months in 2 studies [45,50], and 12 months in 2 studies [45,48].

The loneliness measurement tools used were the UCLA loneliness scale [47,51], which was applied in 4 studies [45,46,50,53], and the De Jong-Gierveld loneliness scale [49,76], which was used in 2 studies [48,54]. Table 2 presents loneliness scores measured in the intervention and control groups, if any, at baseline and follow-ups.

Narrative synthesis showed that there was a reduction in loneliness in the intervention groups at the follow-ups compared with baseline (Table 2). A statistical summary of the loneliness measurements in the intervention and control groups at the follow-ups is reported in the Meta-analysis section. 


\section{Meta-analysis}

We conducted 3 meta-analyses, 1 each for follow-up measurements at 3, 4, and 6 months, involving 3, 2, and 2 studies, respectively.

\section{Meta-analysis for Follow-up at 3 Months}

Three studies [46,50,54] involving 106 participants with follow-up measurements at 3 months were entered into a meta-analysis, which showed a very small reduction in loneliness in favor of the control (SMD 0.02; 95\% CI -0.36 to $0.40)$, but it was not statistically significant $(\mathrm{Z}=0.10 ; P=.92)$. The heterogeneity between studies was not statistically significant $\left(\tau^{2}=0.00 ; \chi_{2}^{2}=0.1 ; P=.95 ; \mathrm{I}^{2}=0 \%\right.$; Figure 2$)$.

Figure 2. Forest plot of standardized mean differences for loneliness at the 3-month follow-up (digital technology intervention vs control).

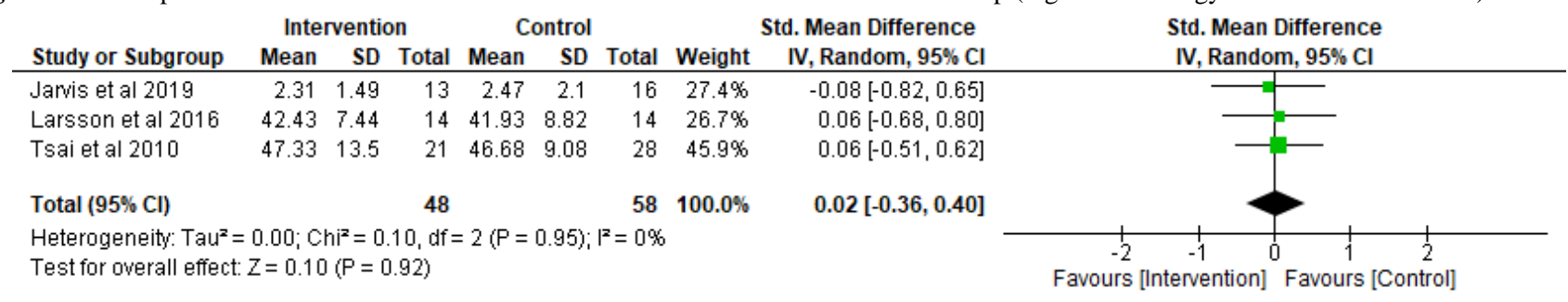

\section{Meta-analysis for Follow-up at 4 Months}

Two studies [53,54] involving 105 participants with 4 month follow-up were entered into a meta-analysis, which revealed a large reduction in loneliness in favor of the intervention (SMD

$-1.11 ; 95 \% \mathrm{CI}-2.60$ to 0.38$)$, but it was not statistically significant $(\mathrm{Z}=1.46 ; P=.14)$. There was a statistically significant high heterogeneity between studies $\left(\tau^{2}=1.03 ; \chi_{1}^{2}=8.8 ; P=.003\right.$; $\mathrm{I}^{2}=88 \%$; Figure 3).

Figure 3. Forest plots of standardized mean differences for loneliness at the 4-month follow-up (digital technology intervention vs control).

\begin{tabular}{|c|c|c|c|c|c|c|c|c|c|}
\hline \multirow{2}{*}{ Study or Subgroup } & \multicolumn{3}{|c|}{ Intervention } & \multicolumn{3}{|c|}{ Control } & \multicolumn{2}{|r|}{ Std. Mean Difference } & \multirow{2}{*}{$\begin{array}{l}\text { Std. Mean Difference } \\
\text { IV, Random, } 95 \% \mathrm{Cl}\end{array}$} \\
\hline & Mean & SD & Total & Mean & SD & Total & Weight & IV, Random, 95\% Cl & \\
\hline Jarvis et al 2019 & 1.38 & 1.33 & 13 & 4 & 1.32 & 16 & $46.6 \%$ & $-1.92[-2.83,-1.02]$ & $-1-$ \\
\hline Morton et al 2018 & 1.86 & 0.66 & 44 & 2.12 & 0.62 & 32 & $53.4 \%$ & $-0.40[-0.86,0.06]$ & \\
\hline Total $(95 \% \mathrm{Cl})$ & & & 57 & & & 48 & $100.0 \%$ & $-1.11[-2.60,0.38]$ & \\
\hline $\begin{array}{l}\text { Heterogeneity: } \operatorname{Tau}^{2}= \\
\text { Test for overall effect: }\end{array}$ & $\begin{array}{l}1.03 ; \mathrm{C} \\
Z=1.46\end{array}$ & $\begin{array}{l}\mathrm{hi}^{2}=8 \\
(\mathrm{P}=\mathrm{l}\end{array}$ & $\begin{array}{l}64, d f= \\
.14)\end{array}$ & $=1(P=$ & $0.003)$ & $i^{2}=88$ & & & $\begin{array}{cccc}1 & 1 & 1 & 1 \\
-4 & -2 & 0 & 2 \\
\text { Favours [Intervention] } & \text { Favours [C }\end{array}$ \\
\hline
\end{tabular}

\section{Meta-analysis for Follow-up at 6 Months}

A meta-analysis involving 2 studies [45,50] with 280 participants with 6 month follow-up showed a very small reduction in loneliness in favor of the intervention (SMD -0.11;
$95 \% \mathrm{CI}-0.54$ to 0.32 ), but it was not statistically significant $(\mathrm{Z}=0.51 ; P=.61)$. There was moderate heterogeneity between studies, but it was not statistically significant $\left(\tau^{2}=0.05 ; \chi_{1}^{2}=1.6\right.$; $P=.21 ; I^{2}=37 \%$; Figure 4$)$.

Figure 4. Forest plots of standardized mean differences for loneliness at the 6-month follow-up (digital technology intervention vs control).

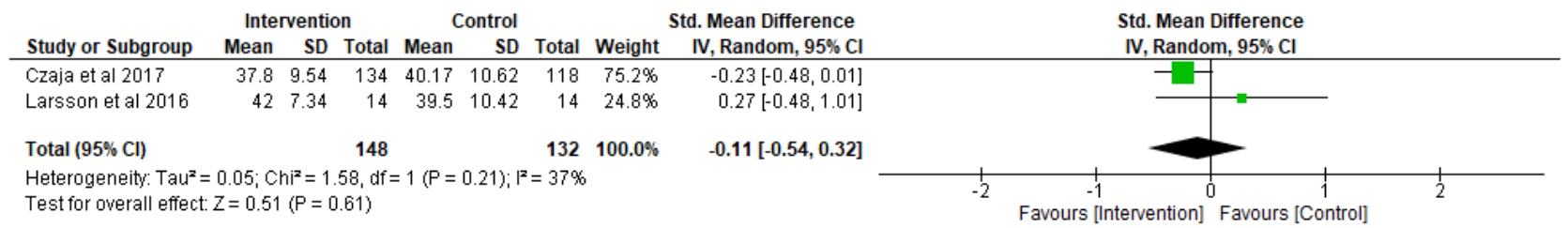

\section{Risk of Bias}

The risk of bias assessment, that is, the risk of bias graph and risk of bias summary are presented in Figure 5 and Figure 6, respectively. A high risk of bias was noted in the attrition bias and other biases; an unclear risk of bias was detected in the blinding of outcome assessment, allocation concealment, and blinding of participants and personnel; and a low risk of bias was observed, especially, in the random sequence generation and selective reporting (Figure 6). In addition, most studies reported only within-group changes and not between-group comparisons of change, which may suggest a weak quality of the reporting of results and the analysis in these studies (Table 2). 
Figure 5. Risk of bias summary. Review authors' judgments about risk of bias in included studies: Czaja et al, 2017 [45], Tsai et al 2010 [46], Larsson et al, 2016 [50], Morton et al, 2018 [53], and Jarvis et al, 2019 [54].

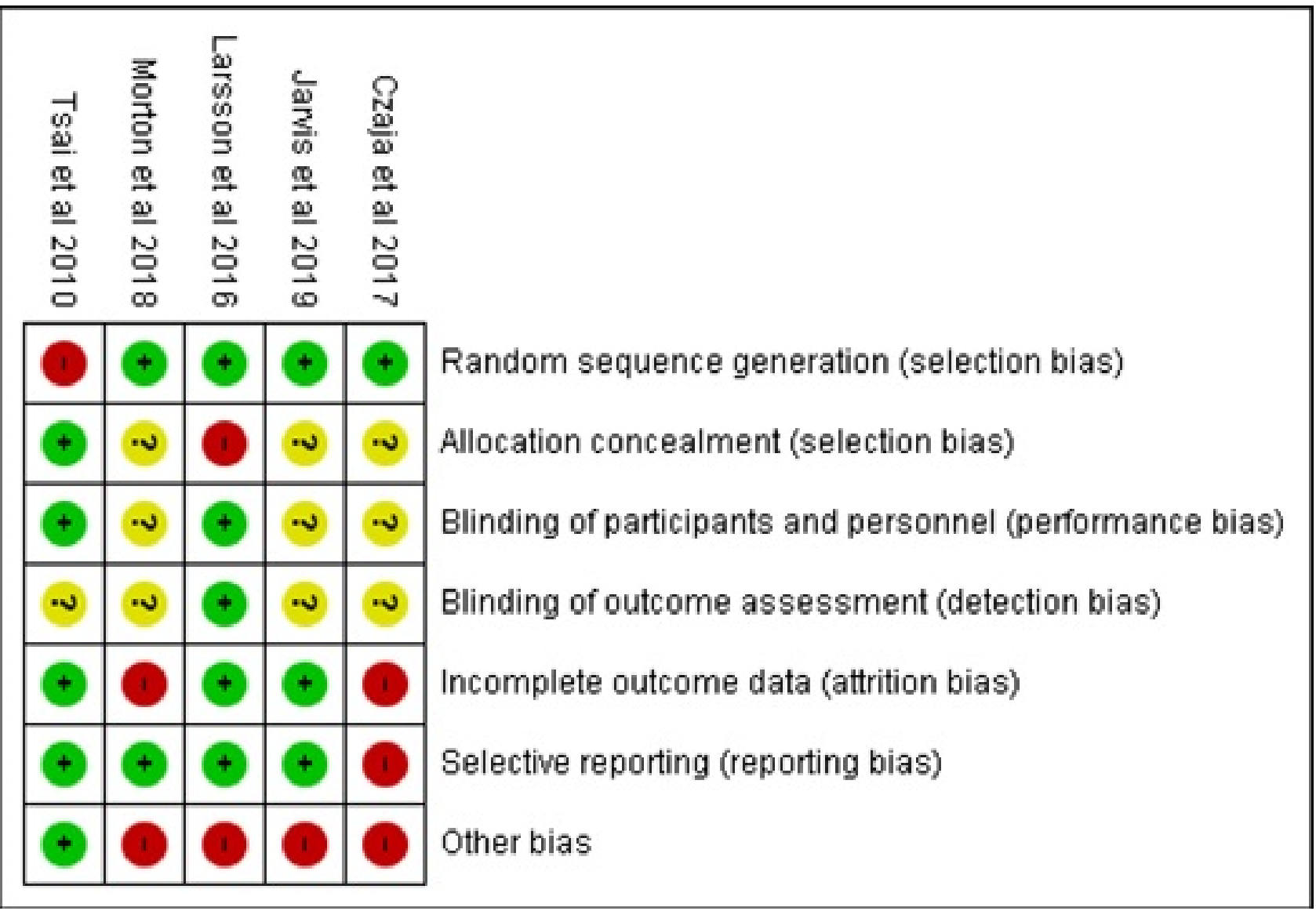

Figure 6. Risk of bias graph. Review authors' judgments about each risk of bias item are presented as percentages across all included studies.

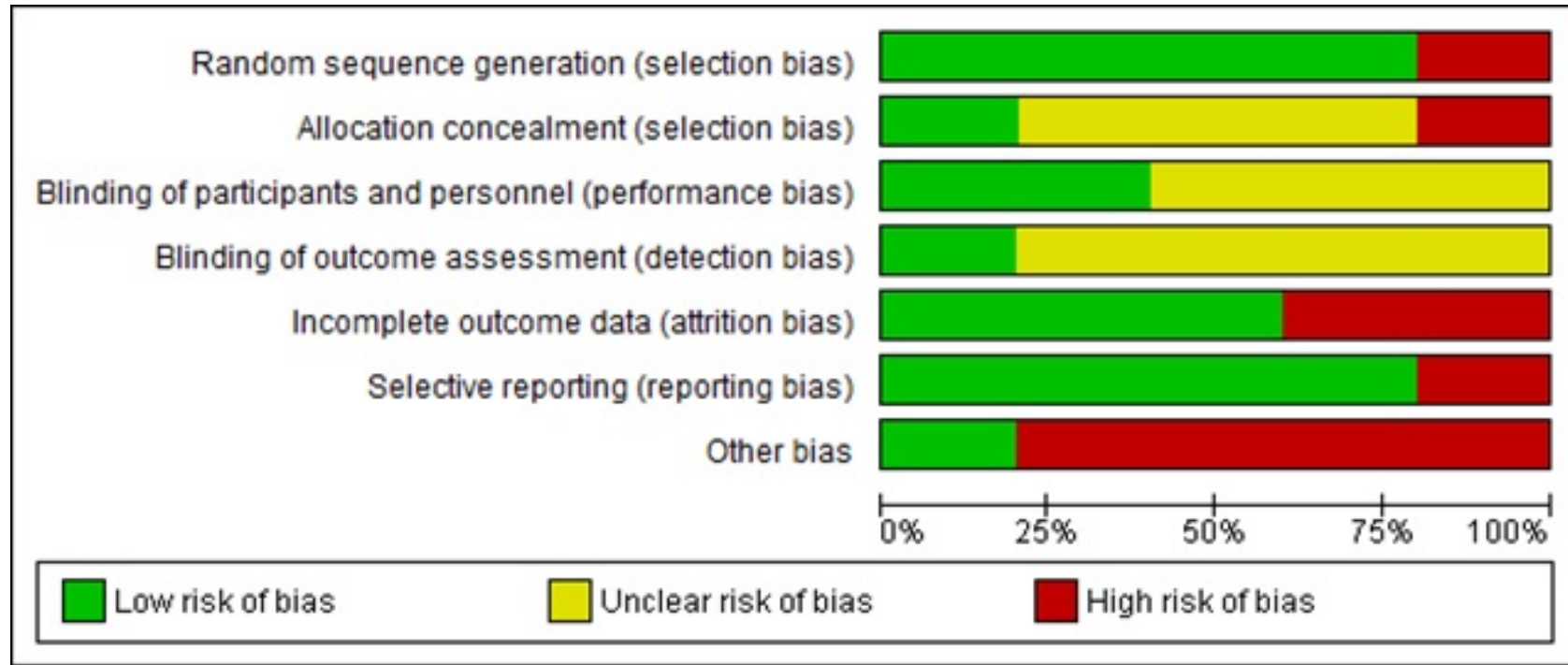

\section{Quality of Evidence}

The quality of evidence was moderate, very low, and moderate in meta-analyses involving 3 [46,50,54], 2 [53,54], and 2 studies
[45,50], with follow-up at 3,4 , and 6 months, respectively (Figure 7). 
Figure 7. GRADE (Grading of Recommendations Assessment, Development and Evaluation) quality of evidence summary. DTI: digital technology intervention; RCT: randomized controlled trial; SMD: standardized mean difference.

\begin{tabular}{|c|c|c|c|c|c|c|c|c|c|}
\hline \multicolumn{10}{|c|}{ Should DTls vs usual care or non-DTls be used for reducing loneliness in adults (Outcome: Loneliness) } \\
\hline \multicolumn{7}{|c|}{ Certainty assessment } & \multicolumn{3}{|c|}{ Summary of findings } \\
\hline \multirow{2}{*}{$\begin{array}{l}\text { Number of } \\
\text { participants } \\
\text { (Number } \\
\text { and type of } \\
\text { studies) }\end{array}$} & \multirow[t]{2}{*}{$\begin{array}{l}\text { Risk of } \\
\text { bias }\end{array}$} & \multirow[t]{2}{*}{ Inconsistency } & \multirow[t]{2}{*}{ Indirectness } & \multirow[t]{2}{*}{ Imprecision } & \multirow[t]{2}{*}{$\begin{array}{l}\text { Publication } \\
\text { bias }\end{array}$} & \multirow{2}{*}{$\begin{array}{l}\text { Overall } \\
\text { certainty of } \\
\text { evidence }\end{array}$} & \multicolumn{2}{|c|}{$\begin{array}{c}\text { Study event rates } \\
(\%)\end{array}$} & \multirow{2}{*}{$\begin{array}{c}\text { Anticipated } \\
\text { absolute effects } \\
\text { Risk difference } \\
\text { with DT Is }\end{array}$} \\
\hline & & & & & & & $\begin{array}{c}\text { With usual } \\
\text { care or } \\
\text { non-DTIs } \\
\end{array}$ & $\begin{array}{l}\text { With } \\
\text { DTIs }\end{array}$ & \\
\hline \multicolumn{10}{|c|}{ Follow up: 3 months } \\
\hline \begin{tabular}{c|}
106 \\
(3 RCTs)
\end{tabular} & $\begin{array}{c}\text { Serious } \\
{[50]}\end{array}$ & Not serious & Not serious & Not serious & None & $\begin{array}{l}\oplus \oplus \oplus \bigcirc \\
\text { Moderate }\end{array}$ & 58 & 48 & $\begin{array}{c}\text { SMD 0.02 SD } \\
\text { higher (0.36 } \\
\text { lower to } 0.4 \\
\text { higher) }\end{array}$ \\
\hline \multicolumn{10}{|c|}{ Follow up: 4 months } \\
\hline \begin{tabular}{c|}
105 \\
(2 RCTs)
\end{tabular} & $\begin{array}{c}\text { Serious } \\
{[53]}\end{array}$ & $\begin{array}{l}\text { Serious } \\
{[53,54]}\end{array}$ & $\begin{array}{l}\text { Serious } \\
{[53,54]}\end{array}$ & $\begin{array}{l}\text { Serious } \\
{[53,54]}\end{array}$ & $\begin{array}{l}\text { Strongly } \\
\text { suspected }\end{array}$ & $\begin{array}{l}\oplus 000 \\
\text { Very low }\end{array}$ & 48 & 57 & $\begin{array}{c}\text { SMD } 1.11 \text { SD } \\
\text { lower } \\
\text { (2.6 lower to } \\
0.38 \text { higher) }\end{array}$ \\
\hline \multicolumn{10}{|c|}{ Follow up: 6 months } \\
\hline \begin{tabular}{c|}
280 \\
(2 RCTs)
\end{tabular} & $\begin{array}{l}\text { Serious } \\
{[45,50]}\end{array}$ & Not serious & Not serious & Not serious & None & $\begin{array}{l}\oplus \oplus \oplus \bigcirc \\
\text { Moderate }\end{array}$ & 132 & 148 & $\begin{array}{l}\text { SMD } 0.11 \text { SD } \\
\text { lower } \\
\text { (0.54 lower to } \\
0.32 \text { higher) }\end{array}$ \\
\hline & & & & rom the estin & ate of effec & & tof the & & $\begin{array}{l}\text { the effect), } \\
\text { of the effect, } \\
\text { true effect }\end{array}$ \\
\hline
\end{tabular}

\section{Discussion}

\section{Principal Findings}

To determine whether DTIs are effective in reducing loneliness in adults, we appraised peer-reviewed empirical research involving the application of DTIs in adults with loneliness. Our systematic review provides a narrative summary (qualitative synthesis) as well as a meta-analysis (statistical synthesis) of the findings. The narrative summary of 6 studies included in our review showed a reduction in loneliness in the intervention groups at follow-up compared with baseline (Table 2). However, our meta-analysis of 5 clinical trials with follow-up measurements at 3, 4, and 6 months showed no statistically significant pooled effect estimates as SMDs, the preferred method for summarizing effects on continuous outcomes such as loneliness. Although not statistically significant, the summary effect size at the 4-month follow-up (Figure 3) was better than the effect size at the 3-month follow-up (Figure 2) and the 6-month follow-up (Figure 4).

Our meta-analysis also revealed that CIs of the summary effects of 2 studies, that is, the studies by Larsson et al [50] and Tsai et al [46], were very wide, and the SMDs from these studies were more in favor of the control group than the intervention group (Figure 2). Thus, the wide range of CIs of the summary effects in these studies leave room for uncertainty about the beneficial effect of DTIs on measures of loneliness.

Overall, the findings of our meta-analysis showed no evidence supporting the effectiveness of DTIs in reducing loneliness in older adults.

\section{Summary of Evidence}

The quality of evidence of the included studies was very low to moderate (Figure 7), and there was a high heterogeneity between studies [53,54] (Figure 2). All the included studies had a high proportion of female participants. Most notably, the total number of participants was low, especially in 2 studies [50,54], and the sample sizes were reduced further due to a high attrition rate in some studies [45,53]. The types and methods of DTIs varied between studies (Table 2), which were conducted in diverse settings (Table 1). Studies were conducted in 6 different countries: the United States, the United Kingdom, Sweden, the Netherlands (these four countries have individualistic cultures), Taiwan, and South Africa (these two countries have collectivist cultures; Table 1). In addition, despite our inclusion criteria of age 18 years and above, the selected studies more commonly involved older people with an average age of 70 years and above.

Loneliness is influenced by culture [77-79], gender [78], and age [5,78], and these factors could have contributed to the pooled estimates being not statistically significant in our meta-analysis. In addition, differences in participants, especially in terms of age, gender, and culture as well as varied types of DTIs, could have contributed to the heterogeneity observed, especially in the meta-analysis with the 4 month follow-up involving 2 studies [53,54], which differed from each other on different parameters, especially the study designs, settings, participants, interventions, and loneliness measurement scales used (Tables 1 and 2).

There are limited published meta-analyses on technological interventions for tackling loneliness, and a few existing studies have covered literature published up to 2009 [28] and 2011 [30]. Our review and meta-analysis included the latest evidence published between January 1, 2010, and July 31, 2019. We did not replicate the findings of earlier meta-analyses that reported evidence suggesting that technological interventions resulted in decreased loneliness $[28,30]$. For example, a meta-analysis 
by Choi et al [30] reported statistically significant evidence suggesting that the internet and computers reduce loneliness. However, they [30] focused on older adults with depression and included the internet and computers only as technological interventions, whereas we included different types of DTIs, and our population of interest was adults of all age groups $(\geq 18$ years). In addition, the meta-analysis by Choi et al [30] included studies $(n=5)$ with different follow-up periods (3-6 months), but they did not report which follow-up measurements were included in their meta-analysis. In our meta-analysis, we conducted separate meta-analyses for measurements at different follow-up periods, that is, 3,4 , and 6 months, as suggested by the Cochrane guidelines [60].

A meta-analysis by Masi et al [28] also reported that technological interventions reduce loneliness, which was more in pre-post studies and nonrandomized studies than in RCTs. However, they included studies with technology and nontechnology-based interventions [28], whereas we focused on studies with DTIs only. In addition, Masi et al [28] did not report how they analyzed measurements at different follow-up periods, whereas we did not combine measurements at different follow-up times, as suggested by the Cochrane guidelines [60]. Nonetheless, Masi et al [28] concluded that technology is yet to be capitalized for loneliness.

Interestingly, our findings provide new insights about DTIs and loneliness. Our meta-analysis showed no statistically significant reduction in loneliness in the intervention groups compared with the control groups at the 3 -, 4-, and 6-month follow-ups. Thus, our findings show no evidence supporting the effectiveness of DTIs in reducing loneliness in older adults, which goes beyond the findings of a recent Cochrane review that reported no evidence of video calls being effective in reducing loneliness in older adults [73].

In addition, our findings refute and contradict a commonly held view that digital technology can solve the problem of loneliness, especially in older people. Nonetheless, digital technologies provide tools and means that facilitate social connection [80], which may help in reducing loneliness for a limited period because the effects of DTIs are short-lived [81]. This may be because digital technologies do not provide real human interaction [80] and cannot replace human contact [45]; thus, they do not reduce social disconnectedness in real life [82] on a long-term basis.

Nonetheless, a review has reported that some nontechnological interventions are effective in reducing loneliness in older people [83], but these interventions require a meta-analytic evaluation. In addition, a recent meta-analysis [84] reported moderate evidence of the effectiveness of a range of social, emotional, and psychological interventions, delivered through technological and nontechnological means, in reducing loneliness in young people aged 3-25 years; however, the analyzed studies had limitations. Therefore, further research is required.

\section{Limitations}

Our study had some limitations: the inclusion of only 6 studies with heterogeneous sets of results and the minimum intervention duration of 3 months, which could have resulted in the inclusion of a small number of studies and possible exclusion of potential studies that would have provided useful evidence.

In addition, we could not conduct subgroup and meta-regression analyses due to the very limited number of studies $(n=5)$ in the meta-analysis and lack of data on loneliness by participants' demographic characteristics. In addition, our study might be narrow because we excluded some studies [44,85-91], which met the technology criterion such as the use of robots, sensors, digital speakers, and apps but did not meet other selection criteria. Thus, our study may be limited to studies about social interactions and connectedness using digital technology tools.

Moreover, another limitation of our review could be the use of a meta-analysis based only on follow-up data. For example, a study by Tsai et al [46] in the 3-month follow-up meta-analysis had an SMD of 0.06 with a $95 \%$ CI of -0.8 to $0.65(P=.03$; Figure 2), which may suggest that these studies may have had higher power to show a difference compared with baseline loneliness.

As recommendations for future research, we suggest that researchers involved in trials agree on a common measure of loneliness and consider reporting of results in a standardized way, which will allow pooling of baseline-adjusted estimates of the treatment effect rather than differences in follow-up means.

\section{Conclusions}

Our meta-analysis showed no evidence supporting the effectiveness of DTIs in reducing loneliness in older adults. Therefore, there is a need for further research involving RCTs [50] with larger sample sizes and longer duration of interventions and follow-up measurement periods. Future research may apply inclusive research designs using a combination of digital apps, including robots, sensors, and social connecting apps, by involving adults aged $45-65$ years, as this segment of the population is more likely to be more technology savvy and digital interventions might be more effective in this age group. Future research might also target ethnic minority communities and specific groups such as lesbian, gay, bisexual, and transgender people where loneliness is common $[8,92]$.

\section{Acknowledgments}

The authors acknowledge support from Liz Callow of Bodleian Health Care Libraries, University of Oxford, for running literature searches. The authors are grateful to Dr Alexandra Farrow (Brunel University London) for checking the first version of the manuscript. This research was funded by the National Institute for Health Research Oxford Biomedical Research Centre (Research Grant IS-BRC-1215-20008). The views expressed are those of the authors and not necessarily those of the NHS, the National Institute for Health Research, or the Department of Health. 


\section{Conflicts of Interest}

None declared.

\section{Multimedia Appendix 1}

Literature searches.

[DOCX File, 37 KB-Multimedia Appendix 1]

\section{References}

1. Lindsay EK, Young S, Brown KW, Smyth JM, Creswell JD. Mindfulness training reduces loneliness and increases social contact in a randomized controlled trial. Proc Natl Acad Sci U S A 2019 Feb 26;116(9):3488-3493 [FREE Full text] [doi: 10.1073/pnas.1813588116] [Medline: $\underline{30808743]}$ ]

2. Loneliness is pervasive and rising, particularly among the young - daily chart. The Economist. 2018. URL: https://www. economist.com/graphic-detail/2018/08/31/loneliness-is-pervasive-and-rising-particularly-among-the-young [accessed 2019-05-03]

3. Mance P. Is Australian experiencing an epidemic of loneliness? Relationships Australia. Canberra, Australia: Relationships Australia National;; 2018 Sep. URL: https://www.relationships.org.au/pdfs/Anepidemicofloneliness20012017.pdf

4. van den Broek T. Gender differences in the correlates of loneliness among Japanese persons aged 50-70. Australas J Ageing 2017 Sep;36(3):234-237. [doi: 10.1111/ajag.12448] [Medline: 28685951]

5. Victor CR, Bowling A. A longitudinal analysis of loneliness among older people in Great Britain. J Psychol 2012;146(3):313-331. [doi: 10.1080/00223980.2011.609572] [Medline: 22574423]

6. Murthy V. Work and the loneliness epidemic. Harv Bus Rev. 2017. URL: https://hbr.org/cover-story/2017/09/ work-and-the-loneliness-epidemic [accessed 2020-10-01]

7. Cohen-Mansfield J, Perach R. Interventions for alleviating loneliness among older persons: a critical review. Am J Health Promot 2015;29(3):109-125. [doi: 10.4278/ajhp.130418-LIT-182] [Medline: 24575725]

8. Jeste DV, Lee EE, Cacioppo S. Battling the modern behavioral epidemic of loneliness: suggestions for research and interventions. JAMA Psychiatry 2020 Jun 01;77(6):553-554. [doi: 10.1001/jamapsychiatry.2020.0027] [Medline: 32129811]

9. Gao Q, Prina M, Prince M, Acosta D, Sosa AL, Guerra M, et al. Loneliness among older adults in Latin America, China, and India: prevalence, correlates and association with mortality. Int J Public Health 2021 Mar 31;66:604449. [doi: 10.3389/ijph.2021.604449]

10. d'Hombres B, Schnepf S, Barjakovà M, Mendonça F. Loneliness - an unequally shared burden in Europe. European Commission. 2018. URL: https://ec.europa.eu/jrc/sites/jrcsh/files/fairness pb2018 loneliness jrc i1.pdf [accessed 2020-09-15]

11. Galea S, Merchant RM, Lurie N. The mental health consequences of COVID-19 and physical distancing: the need for prevention and early intervention. JAMA Intern Med 2020 Jun 1;180(6):817-818. [doi: 10.1001/jamainternmed.2020.1562] [Medline: 32275292]

12. Holmes EA, O'Connor RC, Perry VH, Tracey I, Wessely S, Arseneault L, et al. Multidisciplinary research priorities for the COVID-19 pandemic: a call for action for mental health science. Lancet Psychiatry 2020 Jun;7(6):547-560 [FREE Full text] [doi: 10.1016/S2215-0366(20)30168-1] [Medline: 32304649]

13. Shah SG, Nogueras D, van Woerden HC, Kiparoglou V. The COVID-19 pandemic: a pandemic of lockdown loneliness and the role of digital technology. J Med Internet Res 2020 Nov 05;22(11):e22287 [FREE Full text] [doi: 10.2196/22287] [Medline: $\underline{33108313}$ ]

14. Okwaraji FE, Obiechina KI, Onyebueke GC, Udegbunam ON, Nnadum GS. Loneliness, life satisfaction and psychological distress among out-of-school adolescents in a Nigerian urban city. Psychol Health Med 2018 Oct;23(9):1106-1112. [doi: 10.1080/13548506.2018.1476726] [Medline: 29792068]

15. A Connected Scotland: our strategy for tackling social isolation and loneliness and building stronger social connections. The Scottish Government. 2018. URL: https://www.gov.scot/publications/ connected-scotland-strategy-tackling-social-isolation-loneliness-building-stronger-social-connections/pages/6/ [accessed 2019-05-03]

16. Qualter P. Loneliness in children and adolescents: what do schools and teachers need to know and how can they help? Pastoral Care in Ed 2003 Jun;21(2):10-18. [doi: 10.1111/1468-0122.00257]

17. Cohen-Mansfield J, Hazan H, Lerman Y, Shalom V. Correlates and predictors of loneliness in older-adults: a review of quantitative results informed by qualitative insights. Int Psychogeriatr 2016 Apr;28(4):557-576. [doi: 10.1017/S1041610215001532] [Medline: 26424033]

18. Deckx L, van den Akker M, Buntinx F. Risk factors for loneliness in patients with cancer: a systematic literature review and meta-analysis. Eur J Oncol Nurs 2014 Oct;18(5):466-477. [doi: 10.1016/j.ejon.2014.05.002] [Medline: 24993076]

19. Chen Y, Hicks A, While AE. Loneliness and social support of older people in China: a systematic literature review. Health Soc Care Community 2014 Mar;22(2):113-123. [doi: 10.1111/hsc.12051] [Medline: 23714357] 
20. Rico-Uribe LA, Caballero FF, Martín-María N, Cabello M, Ayuso-Mateos JL, Miret M. Association of loneliness with all-cause mortality: a meta-analysis. PLoS One 2018 Jan 4;13(1):e0190033 [FREE Full text] [doi: 10.1371/journal.pone.0190033] [Medline: 29300743]

21. Holt-Lunstad J. The potential public health relevance of social isolation and loneliness: prevalence, epidemiology, and risk factors. Public Policy Aging Rep 2017;27(4):127-130. [doi: 10.1093/ppar/prx030]

22. Holt-Lunstad J, Smith TB, Baker M, Harris T, Stephenson D. Loneliness and social isolation as risk factors for mortality: a meta-analytic review. Perspect Psychol Sci 2015 Mar;10(2):227-237. [doi: 10.1177/1745691614568352] [Medline: 25910392]

23. Deckx L, van den Akker M, Buntinx F, van Driel M. A systematic literature review on the association between loneliness and coping strategies. Psychol Health Med 2018 Sep;23(8):899-916. [doi: 10.1080/13548506.2018.1446096] [Medline: 29533084]

24. Lara E, Martín-María N, De la Torre-Luque A, Koyanagi A, Vancampfort D, Izquierdo A, et al. Does loneliness contribute to mild cognitive impairment and dementia? A systematic review and meta-analysis of longitudinal studies. Ageing Res Rev 2019 Jul;52:7-16. [doi: 10.1016/j.arr.2019.03.002] [Medline: 30914351]

25. Peytrignet S, Garforth-Bles S, Keohane K. Loneliness monetisation report : analysis for the Department for Digital, Culture, Media \& Sport. 2020. URL: https://assets.publishing.service.gov.uk/government/uploads/system/uploads/attachment_data/ file/910789/Loneliness monetisation report.pdf [accessed 2021-05-13]

26. Jeffrey K, Abdallah S, Michaelson J. The cost of loneliness to UK employers. New Economics Foundation. 2017. URL: https://neweconomics.org/2017/02/cost-loneliness-uk-employers/2017 [accessed 2020-05-20]

27. Steptoe A, Shankar A, Demakakos P, Wardle J. Social isolation, loneliness, and all-cause mortality in older men and women. Proc Natl Acad Sci U S A 2013 Apr 9;110(15):5797-5801 [FREE Full text] [doi: 10.1073/pnas.1219686110] [Medline: 23530191]

28. Masi CM, Chen H, Hawkley LC, Cacioppo JT. A meta-analysis of interventions to reduce loneliness. Pers Soc Psychol Rev 2011 Aug;15(3):219-266 [FREE Full text] [doi: 10.1177/1088868310377394] [Medline: 20716644]

29. Pu L, Moyle W, Jones C, Todorovic M. The effectiveness of social robots for older adults: a systematic review and meta-analysis of randomized controlled studies. Gerontologist 2019 Jan 09;59(1):37-51. [doi: 10.1093/geront/gny046] [Medline: 29897445]

30. Choi M, Kong S, Jung D. Computer and internet interventions for loneliness and depression in older adults: a meta-analysis. Healthc Inform Res 2012 Sep;18(3):191-198 [FREE Full text] [doi: 10.4258/hir.2012.18.3.191] [Medline: 23115742]

31. Morris ME, Adair B, Miller K, Ozanne E, Hansen R, Pearce AJ, et al. Smart-Home Technologies to Assist Older People to Live Well at Home. Aging Sci 2013;01(01):1-9. [doi: 10.4172/2329-8847.1000101]

32. Morris ME, Adair B, Ozanne E, Kurowski W, Miller KJ, Pearce AJ, et al. Smart technologies to enhance social connectedness in older people who live at home. Australas J Ageing 2014 Sep;33(3):142-152. [doi: 10.1111/ajag.12154] [Medline: 24730370]

33. Pearce AJ, Adair B, Miller K, Ozanne E, Said C, Santamaria N, et al. Robotics to enable older adults to remain living at home. J Aging Res 2012 Dec 4;2012:538169 [FREE Full text] [doi: 10.1155/2012/538169] [Medline: 23304507]

34. Hagan R, Manktelow R, Taylor BJ, Mallett J. Reducing loneliness amongst older people: a systematic search and narrative review. Aging Ment Health 2014 Jan 17;18(6):683-693. [doi: 10.1080/13607863.2013.875122] [Medline: 24437736]

35. Miller KJ, Adair BS, Pearce AJ, Said CM, Ozanne E, Morris MM. Effectiveness and feasibility of virtual reality and gaming system use at home by older adults for enabling physical activity to improve health-related domains: a systematic review. Age Ageing 2014 Mar;43(2):188-195. [doi: 10.1093/ageing/aft194] [Medline: 24351549]

36. Medical Advisory Secretariat. Social isolation in community-dwelling seniors: an evidence-based analysis. Ont Health Technol Assess Ser 2008;8(5):1-49 [FREE Full text] [Medline: 23074510]

37. Shah SG, Nogueras D, van Woerden H, Kiparoglou V. Effectiveness of digital technology interventions to reduce loneliness in adults: a protocol for a systematic review and meta-analysis. BMJ Open 2019 Sep 27;9(9):e032455 [FREE Full text] [doi: 10.1136/bmjopen-2019-032455] [Medline: 31562164]

38. Higgins J, Thomas J, Chandler J, Cumpston M, Deeks J, Clarke M. Chapter I: Introduction. Cochrane Handbook for Systematic Reviews of Interventions Version 6.1 (Updated September 2020). 2020. URL: https://training.cochrane.org/ handbook/archive/v6.1/chapter-i [accessed 2020-09-17]

39. Moher D, Liberati A, Tetzlaff J, Altman DG, PRISMA Group. Preferred reporting items for systematic reviews and meta-analyses: the PRISMA statement. Br Med J 2009 Jul 21;339:b2535 [FREE Full text] [doi: 10.1136/bmj.b2535] [Medline: 19622551$]$

40. Shah S, Nogueras D, van Woerden H, Kiparoglou V. The effectiveness of digital technology interventions to reduce loneliness in adult people: a systematic review and meta-analysis. PROSPERO 2019 CRD42019131524. 2019. URL: https:/ /Www.crd.york.ac.uk/PROSPERO/display_record.php?RecordID=131524 [accessed 2019-06-10]

41. What are some everyday examples of digital technology? Quora. URL: https://www.quora.com/What-are-digital-technologies [accessed 2019-06-06] 
42. Hemberg J, Fischer RS. A window toward the world: older adults' experiences of becoming in health and developing as human beings through interacting with others using real video communication. Holist Nurs Pract 2018;32(2):90-97. [doi: 10.1097/HNP.0000000000000254] [Medline: 29432355]

43. Gao Q, Ebert D, Chen X, Ding Y. Design of a mobile social community platform for older Chinese people in urban areas. Hum Factors Man 2012 Jun 27;25(1):66-89. [doi: 10.1002/hfm.20523]

44. Austin J, Dodge HH, Riley T, Jacobs PG, Thielke S, Kaye J. A smart-home system to unobtrusively and continuously assess loneliness in older adults. IEEE J Transl Eng Health Med 2016 Jun 10;4:1-11. [doi: 10.1109/jtehm.2016.2579638]

45. Czaja SJ, Boot WR, Charness N, Rogers WA, Sharit J. Improving social support for older adults through technology: findings from the PRISM randomized controlled trial. Gerontologist 2018 May 8;58(3):467-477 [FREE Full text] [doi: 10.1093/geront/gnw249] [Medline: 28201730]

46. Tsai H, Tsai Y, Wang H, Chang Y, Chu HH. Videoconference program enhances social support, loneliness, and depressive status of elderly nursing home residents. Aging Ment Health 2010 Nov;14(8):947-954. [doi: 10.1080/13607863.2010.501057] [Medline: 21069600]

47. Russell D, Peplau LA, Cutrona CE. The revised UCLA Loneliness Scale: concurrent and discriminant validity evidence. J Pers Soc Psychol 1980 Sep;39(3):472-480. [Medline: 7431205]

48. van der Heide LA, Willems CG, Spreeuwenberg MD, Rietman J, de Witte LP. Implementation of CareTV in care for the elderly: the effects on feelings of loneliness and safety and future challenges. Technol Disab 2012 Dec 12;24(4):283-291. [doi: $10.3233 /$ tad-120359]

49. Gierveld JD, Tilburg TV. A 6-item scale for overall, emotional, and social loneliness. Res Aging 2006 Sep 11;28(5):582-598. [doi: $10.1177 / 0164027506289723$ ]

50. Larsson E, Padyab M, Larsson-Lund M, Nilsson I. Effects of a social internet-based intervention programme for older adults: an explorative randomised crossover study. Br J Occup Ther 2016 Jul 19;79(10):629-636. [doi: 10.1177/0308022616641701]

51. Russell DW. UCLA Loneliness Scale (Version 3): reliability, validity, and factor structure. J Pers Assess 1996 Feb;66(1):20-40. [doi: 10.1207/s15327752jpa6601_2] [Medline: 8576833]

52. Engelberg E, Sjöberg L. Emotional intelligence and inter-personal skills. In: Schulze R, Roberts RD, editors. Emotional Intelligence: An International Handbook. Cambridge, MA: Hogrefe \& Huber; 2005:289-305.

53. Morton TA, Wilson N, Haslam C, Birney M, Kingston R, McCloskey L. Activating and guiding the engagement of seniors with online social networking. J Aging Health 2018 Jan;30(1):27-51. [doi: 10.1177/0898264316664440] [Medline: 27530332]

54. Jarvis MA, Padmanabhanunni A, Chipps J. An evaluation of a low-intensity cognitive behavioral therapy mHealth-supported intervention to reduce loneliness in older people. Int J Environ Res Public Health 2019 Apr 11;16(7):1305 [FREE Full text] [doi: 10.3390/ijerph16071305] [Medline: 30979042]

55. Shamseer L, Moher D, Clarke M, Ghersi D, Liberati A, Petticrew M, PRISMA-P Group. Preferred reporting items for systematic review and meta-analysis protocols (PRISMA-P) 2015: elaboration and explanation. Br Med J 2015 Jan 02;350:g7647 [FREE Full text] [doi: 10.1136/bmj.g7647] [Medline: 25555855]

56. Munn Z, Tufanaru C, Aromataris E. JBI's systematic reviews: data extraction and synthesis. Am J Nurs 2014 Jul;114(7):49-54. [doi: 10.1097/01.NAJ.0000451683.66447.89] [Medline: 25742352]

57. Munn Z, Stern C, Aromataris E, Lockwood C, Jordan Z. What kind of systematic review should I conduct? A proposed typology and guidance for systematic reviewers in the medical and health sciences. BMC Med Res Methodol 2018 Jan 10;18:5 [FREE Full text] [doi: 10.1186/s12874-017-0468-4] [Medline: 29316881]

58. Larsson E, Nilsson I, Lund ML. Participation in social internet-based activities: five seniors' intervention processes. Scand J Occup Ther 2013 Nov 30;20(6):471-480. [doi: 10.3109/11038128.2013.839001] [Medline: 24074300]

59. Tufanaru C, Munn Z, Aromataris E, Campbell J, Hopp L. Chapter 3: Systematic reviews of effectiveness. In: Aromataris E, Munn Z, editors. JBI Manual for Evidence Synthesis. Adelaide: Joanna Briggs Institute (JBI); 2020. [doi: 10.46658/JBIMES-20-04]

60. Higgins J, Green S. Cochrane handbook for systematic reviews of interventions - Version 5.1.0. The Cochrane Collaboration. 2011. URL: https://handbook-5-1.cochrane.org/front page.htm [accessed 2019-05-13]

61. Takeshima N, Sozu T, Tajika A, Ogawa Y, Hayasaka Y, Furukawa TA. Which is more generalizable, powerful and interpretable in meta-analyses, mean difference or standardized mean difference? BMC Med Res Methodol 2014 Feb 21;14:30 [FREE Full text] [doi: 10.1186/1471-2288-14-30] [Medline: 24559167]

62. Valtorta NK, Kanaan M, Gilbody S, Hanratty B. Loneliness, social isolation and social relationships: what are we measuring? A novel framework for classifying and comparing tools. BMJ Open 2016 Apr 18;6(4):e010799 [FREE Full text] [doi: 10.1136/bmjopen-2015-010799] [Medline: 27091822]

63. de Jong-Gierveld J, Kamphuls F. The development of a Rasch-Type Loneliness Scale. Appl Psychol Meas 2016 Jul 27;9(3):289-299. [doi: 10.1177/014662168500900307]

64. Eccleston C, Fisher E, Craig L, Duggan GB, Rosser BA, Keogh E. Psychological therapies (internet-delivered) for the management of chronic pain in adults. Cochrane Database Syst Rev 2014 Feb 26;2014(2):CD010152. [doi:

10.1002/14651858.CD010152.pub2] [Medline: 24574082] 
65. Grimshaw J, Campbell M, Eccles M, Steen N. Experimental and quasi-experimental designs for evaluating guideline implementation strategies. Fam Pract 2000 Feb;17(Suppl 1):S11-S16. [doi: 10.1093/fampra/17.suppl 1.s11] [Medline: 10735262]

66. Review Manager (RevMan) Version 5. The Cochrane Collaboration. URL: https://community.cochrane.org/help/ tools-and-software/revman-5 [accessed 2019-07-19]

67. Borenstein M, Hedges L, Higgins J, Rothstein H. Introduction to Meta-Analysis. Hoboken, New Jersey, United States: John Wiley \& Sons; 2009:1-452.

68. Borenstein M, Hedges LV, Higgins JP, Rothstein HR. A basic introduction to fixed-effect and random-effects models for meta-analysis. Res Synth Methods 2010 Apr 21;1(2):97-111. [doi: 10.1002/jrsm.12] [Medline: 26061376]

69. da Costa BR, Juni P. Systematic reviews and meta-analyses of randomized trials: principles and pitfalls. Eur Heart J 2014 Dec 14;35(47):3336-3345. [doi: 10.1093/eurheartj/ehu424] [Medline: 25416325]

70. Guyatt GH, Oxman AD, Sultan S, Glasziou P, Akl EA, Alonso-Coello P, et al. GRADE guidelines: 9. Rating up the quality of evidence. J Clin Epidemiol 2011 Dec;64(12):1311-1316. [doi: 10.1016/j.jclinepi.2011.06.004] [Medline: 21802902]

71. Sedgwick P. Meta-analyses: how to read a funnel plot. Br Med J 2013 Mar 01;346(mar01 2):f1342. [doi: 10.1136/bmj.f1342]

72. Sutton A, Abrams K, Jones D, Sheldon T, Song F. Methods for Meta-Analysis in Medical Research. Hoboken, New Jersey, United States: John Wiley \& Sons; 2000:1-348.

73. Noone C, McSharry J, Smalle M, Burns A, Dwan K, Devane D, et al. Video calls for reducing social isolation and loneliness in older people: a rapid review. Cochrane Database Syst Rev 2020 May 21;5(5):CD013632. [doi: 10.1002/14651858.CD013632] [Medline: 32441330]

74. Higgins J, Thomas J, Chandler J, Cumpston M, Li T, Page M, et al, editors. Cochrane Handbook for Systematic Reviews of Interventions. Hoboken, New Jersey, United States: Wiley-Blackwell; 2019:1-728.

75. Higgins JP, Thompson SG, Deeks JJ, Altman DG. Measuring inconsistency in meta-analyses. Br Med J 2003 Sep 6;327(7414):557-560 [FREE Full text] [doi: 10.1136/bmj.327.7414.557] [Medline: 12958120]

76. Gierveld JD, Van Tilburg T. The De Jong Gierveld short scales for emotional and social loneliness: tested on data from 7 countries in the UN generations and gender surveys. Eur J Ageing 2010 Jun;7(2):121-130 [FREE Full text] [doi: 10.1007/s10433-010-0144-6] [Medline: 20730083]

77. van Staden WC, Coetzee K. Conceptual relations between loneliness and culture. Curr Opin Psychiatry 2010 Nov;23(6):524-529. [doi: 10.1097/YCO.0b013e32833f2ff9] [Medline: 20838346]

78. Barreto M, Victor C, Hammond C, Eccles A, Richins MT, Qualter P. Loneliness around the world: Age, gender, and cultural differences in loneliness. Pers Individ Dif 2021 Feb 01;169:110066 [FREE Full text] [doi: 10.1016/j.paid.2020.110066] [Medline: 33536694]

79. Heu LC, van Zomeren M, Hansen N. Does loneliness thrive in relational freedom or restriction? The culture-loneliness framework. Rev Gen Psychol 2020 Sep 24;25(1):60-72. [doi: 10.1177/1089268020959033]

80. Anderson G, Thayer C. A national survey of adults 45 and older: loneliness and social connections. AARP Foundation, Washington DC. 2018. URL: https://www.aarp.org/content/dam/aarp/research/surveys statistics/life-leisure/2018/ loneliness-social-connections-2018.doi.10.26419-2Fres.00246.001.pdf [accessed 2020-09-21]

81. Chen YR, Schulz PJ. The effect of information communication technology interventions on reducing social isolation in the elderly: a systematic review. J Med Internet Res 2016 Jan 28;18(1):e18 [FREE Full text] [doi: 10.2196/jmir.4596] [Medline: 26822073]

82. Kushlev K, Proulx JD, Dunn EW. Digitally connected, socially disconnected: the effects of relying on technology rather than other people. Comput Hum Behav 2017 Nov;76:68-74. [doi: 10.1016/j.chb.2017.07.001]

83. Poscia A, Stojanovic J, La MD, Duplaga M, Grysztar M, Moscato U, et al. Interventions targeting loneliness and social isolation among the older people: an update systematic review. Exp Gerontol 2018 Feb;102:133-144. [doi: 10.1016/j.exger.2017.11.017] [Medline: 29199121]

84. Eccles AM, Qualter P. Review: Alleviating loneliness in young people - a meta-analysis of interventions. Child Adolesc Ment Health 2021 Feb;26(1):17-33. [doi: 10.1111/camh.12389] [Medline: 32406165]

85. Ballantyne A, Trenwith L, Zubrinich S, Corlis M. 'I feel less lonely': what older people say about participating in a social networking website. Qual Ageing Older Adults 2010 Sep 13;11(3):25-35. [doi: 10.5042/qiaoa.2010.0526]

86. Kahlbaugh PE, Sperandio AJ, Carlson AL, Hauselt J. Effects of playing wii on well-being in the elderly: physical activity, loneliness, and mood. Act Adapt Aging 2011 Oct;35(4):331-344. [doi: 10.1080/01924788.2011.625218]

87. Blažun H, Saranto K, Rissanen S. Impact of computer training courses on reduction of loneliness of older people in Finland and Slovenia. Comput Hum Behav 2012 Jul;28(4):1202-1212. [doi: 10.1016/j.chb.2012.02.004]

88. Blusi M, Asplund K, Jong M. Older family carers in rural areas: experiences from using caregiver support services based on Information and Communication Technology (ICT). Eur J Ageing 2013 Sep;10(3):191-199 [FREE Full text] [doi: 10.1007/s10433-013-0260-1] [Medline: 28804294]

89. Chopik WJ. The benefits of social technology use among older adults are mediated by reduced loneliness. Cyberpsychol Behav Soc Netw 2016 Sep;19(9):551-556 [FREE Full text] [doi: 10.1089/cyber.2016.0151] [Medline: 27541746]

90. Siniscarco MT, Love-Williams C, Burnett-Wolle S. Video conferencing: an intervention for emotional loneliness in long-term care. Act Adapt Aging 2017 Jun 15;41(4):316-329. [doi: 10.1080/01924788.2017.1326763] 
91. Barbosa NB, Franz R, Judges R, Beermann C, Baecker R. Can digital technology enhance social connectedness among older adults? A feasibility study. J Appl Gerontol 2019 Jan;38(1):49-72. [doi: 10.1177/0733464817741369] [Medline: 29166818]

92. Hughes M. Loneliness and social support among lesbian, gay, bisexual, transgender and intersex people aged 50 and over. Ageing Soc 2015 Jul 16;36(9):1961-1981. [doi: 10.1017/s0144686x1500080x]

\section{Abbreviations}

DTI: digital technology intervention

GRADE: Grading of Recommendations Assessment, Development and Evaluation

PICO: participant, intervention, comparator, and outcome

PRISMA: Preferred Reporting Items for Systematic Reviews and Meta-Analyses

RCT: randomized controlled trial

SMD: standardized mean difference

UCLA: University of California, Los Angeles

Edited by R Kukafka; submitted 01.10.20; peer-reviewed by L Jordan, M Ji, J Hwang; comments to author 27.10.20; revised version received 09.11.20; accepted 19.04.21; published 04.06.21

Please cite as:

Shah SGS, Nogueras D, van Woerden HC, Kiparoglou V

Evaluation of the Effectiveness of Digital Technology Interventions to Reduce Loneliness in Older Adults: Systematic Review and Meta-analysis

J Med Internet Res 2021;23(6):e24712

URL: https://www.jmir.org/2021/6/e24712

doi: $10.2196 / 24712$

PMID:

(CSyed Ghulam Sarwar Shah, David Nogueras, Hugo Cornelis van Woerden, Vasiliki Kiparoglou. Originally published in the Journal of Medical Internet Research (https://www.jmir.org), 04.06.2021. This is an open-access article distributed under the terms of the Creative Commons Attribution License (https://creativecommons.org/licenses/by/4.0/), which permits unrestricted use, distribution, and reproduction in any medium, provided the original work, first published in the Journal of Medical Internet Research, is properly cited. The complete bibliographic information, a link to the original publication on https://www.jmir.org/, as well as this copyright and license information must be included. 